\title{
The role of heterogeneity of patients' preferences in kidney transplantation
}

\author{
Mesfin G. Genie ${ }^{\mathrm{a}, \mathrm{e}}$, Antonio Nicoló ${ }^{\mathrm{b}, \mathrm{c}}$, Giacomo Pasini ${ }^{\mathrm{a}, \mathrm{d}, *}$ \\ a Dipartimento di Economia, Università Ca' Foscari di Venezia, Cannaregio 873, Fondamenta San Giobbe, 30121 Venezia, Italy \\ b Dipartimento di Scienze Economiche “Marco Fanno", Università degli Studi di Padova, Via del Santo 33, 35123 Padova, Italy \\ ' School of Social Sciences, The University of Manchester, Manchester M13 9PL, UK \\ d NETSPAR, Network for Studies on Pensions, Ageing and Retirement, Tilburg, The Netherlands \\ e Health Economics Research Unit, University of Aberdeen, Aberdeen AB25 2QN, UK
}

\section{A R T I C L E I N F O}

\section{Article history:}

Received 29 July 2019

Received in revised form 23 April 2020

Accepted 24 April 2020

Available online 16 May 2020

\section{JEL classification:}

C25

C90

D61

I18

Keywords:

Discrete choice experiment

Mixed logit

Willingness to wait

Marginal kidney

\begin{abstract}
A B S T R A C T
We elicit time and risk preferences for kidney transplantation from the entire population of patients of the largest Italian transplant centre using a discrete choice experiment (DCE). We measure patients' willingness-to-wait (WTW) for receiving a kidney with one-year longer expected graft survival, or a low risk of complication. Using a mixed logit in WTW-space model, we find heterogeneity in patients' preferences. Our model allows WTW to vary with patients' age and duration of dialysis. The results suggest that WTW correlates with age and duration of dialysis, and that accounting for patients' preferences in the design of kidney allocation protocols could increase their welfare. The implication for transplant practice is that eliciting patients' preferences could help in the allocation of "non-ideal" kidneys.
\end{abstract}

(c) 2020 The Authors. Published by Elsevier B.V. This is an open access article under the CC BY-NC-ND license (http://creativecommons.org/licenses/by-nc-nd/4.0/).

\section{Introduction}

Kidney transplantation carries several advantages over dialysis treatment for patients with end-stage renal disease (ESRD) in terms of long-term mortality risk, improved survival rates and quality of life (Merion et al., 2005; Held et al., 2016). Nevertheless, the disparity between the large number of transplant candidates and the scarcity of organs available continues to increase. There are currently 6372 patients waiting for a kidney transplant in Italy, and more than 2500 of them have been waiting for more than three

* Corresponding author at: Dipartimento di Economia, Università Ca' Foscari di Venezia, Cannaregio 873, Fondamenta San Giobbe, 30121 Venezia, Italy. years. ${ }^{1}$ Recent data in both the US and Europe confirm that the demand for kidneys far outpaces supply (Hart et al., 2018), prompting physicians to push the limits of donor suitability to utilise organs from donors with characteristics different from the "ideal" situation. Selection criteria for donor appropriateness have been widened significantly in recent years to include older persons and those with co-morbidities such as hypertension, diabetes, suboptimal renal function, or risky behaviours that could potentially increase the risk of infectious disease transmission (the socalled Expanded Criteria Donors, ECD). ${ }^{2}$ As a consequence,

\footnotetext{
1 Based on the Italian Ministry of Health data as of December 31, 2019.

2 Being precise, ECD are deceased donor kidneys conveying a 70\% or higher risk for a graft loss for transplant recipients relative to the ideal
} 
an increasing number of transplants are now performed by expanding the pool of donors to include those who would have been considered unsuitable before. The ECD program implemented since 2002 in the US and the Eurotransplant Seniors Program (ESP) implemented since 1999 in Europe are two examples of such policies.

The result of kidney transplantation from marginal donors is one of the most topical issues in the transplant literature (examples include Ojo et al., 2001; Metzger et al., 2003; Merion et al., 2005 and more recently Sunjae Bae et al., 2019). From a clinical point of view, ECD or "marginal" kidneys, while inferior to standard criteria donor (SCD) kidneys, may prolong the life of the recipient compared to dialysis treatment. Moreover, transplantation with a marginal donor kidney is more cost-effective than dialysis as a means of treating ESRD (Held et al., 2016; Eggers, 1992; Eggers and Kucken, 1994).

The functional recovery following a transplant crucially depends on the length of the cold ischemia time, defined as the interval between the procurement of the organ and its reperfusion during the recipient operation. Since kidneys begin to degrade during this cold ischemia time, surgeons typically transplant them within $24 \mathrm{~h}$. If patients' preferences were known in advance and ECD organs were offered only to patients who are willing to accept them, the number of organs discarded could be substantially reduced for two reasons: firstly, the chances that the patient does not accept an ECD kidney, if offered, are smaller. Secondly, it may well be that the pool of patients willing to receive an ECD organ is larger than expected. Patients' preferences, however, are largely ignored in kidney allocation algorithms. This is true for any organ transplant, but, while in the case of other organs (e.g., liver, heart, and lung) alternative options are considerably limited, dialysis could be a reasonable option against which patients on the waiting list can balance risks and benefits. As a result, different patients may have heterogeneous preferences regarding the proposed treatment: they may prefer to wait longer with the prospect of receiving an "ideal" kidney, or they may be willing to accept an organ of inferior quality with the advantage of shorter waiting time. Preferences may or may not correlate with recipients' social, cultural, or economic status and psychological predispositions.

There is a limited but growing body of literature on ESRD patients' preferences. A recent paper by Agarwal et al. (2019) establishes an empirical framework to analyse how trade-offs embedded in waitlist systems map into individual preferences and applies it to the allocation of deceased donor kidneys. The researchers develop a method for estimating patient preferences using administrative data and apply it to the kidney waitlist data from New York to estimate payoffs from various types of transplants. Reese et al. (2010) assessed patients' willingness to accept a kidney from a donor with an increased risk of blood-borne viral infection (DIRVI) in the USA, and Kamran et al. (2017)

donation and are characterised by a donor age older than 60 years or older than 50 years and accompanied by two additional risk factors, including a history of hypertension, elevated terminal donor creatinine, and cerebrovascular cause of death (Metzger et al., 2003). employed a discrete choice experiment (DCE) to evaluate patients' willingness to accept a marginal graft. ${ }^{3}$

We contribute to this literature by eliciting preferences of the entire population of patients waiting for a transplant at the largest transplant centre in Italy; the Pancreas and Kidney Transplant Unit of the School of Medicine, University of Padova. We used a DCE to investigate patients' preferences for time and risk attributes of kidney transplantation and examine trade-offs for these attributes based on a willingness-to-wait (WTW) approach. DCEs in health economics are typically administered to a sample drawn from the general public. However, as these individuals are unlikely to be familiar with the disease and treatments being researched, and may never face such a disease in their lifetime, we instead used real patients from the waiting list. This allowed us to minimise the chances of misunderstanding the transplant alternatives used in the experiment

Given the specific topic of the paper, having real patients rather than a sample from the general public is even more important: this is precisely the subpopulation who would be affected by any change in the ECD organs allocation protocol. We find a significant WTW heterogeneity for all the attributes in the experiment. Moreover, WTW correlates with patients' age and duration of dialysis. Since reducing cold ischemia time and reducing organ waste are important design objectives for every kidney allocation scheme, our findings have important implications for the design of efficient kidney allocation algorithms.

The rest of this article is organised as follows. Section 2 provides background information about the Italian Transplant Network, the Pancreas and Kidney Transplant Unit of the School of Medicine of the University of Padova, where we run our experiment, and the subjects involved in the study. Section 3 describes the design of the experiment. Section 4 describes our modelling approach. Section 5 presents the model results. Section 6 provides a simulation exercise to show how much a preference-based allocation protocol would change the outcomes of interest. Section 7 discusses implications for transplant practice and draws conclusions.

\section{The Italian transplant network}

In Italy, transplantation is an intervention that falls within the essential levels of assistance (LEA), i.e., those medical treatments that the Italian National Healthcare System (NHS) is required to provide free of charge to every resident. For patients who suffer from ESRD, all medical treatments, including dialysis and kidney transplant, are provided free of charge. There are 42 kidney transplant centres in Italy. A transplant centre is suggested to each ESRD patient who is declared suitable for a kidney transplant, which typically is the centre nearest to the patient's residence. A transplant candidate can also choose to enrol at any other centre provided there is an available slot: each

\footnotetext{
3 For a systematic review of discrete choice experiments and conjoint analysis studies measuring trade-offs in nephrology, look at Clark et al. (2018).
} 
Table 1

Attributes and levels used to define the kidney transplant choices.

\begin{tabular}{ll}
\hline Attributes & Definition \\
\hline $\begin{array}{l}\text { Waiting time } \\
\text { Graft survival }\end{array}$ & $\begin{array}{l}\text { The number of months one has to wait to obtain the proposed transplant } \\
\text { The expected length of time the kidney functions well enough to keep recipients from either } \\
\text { needing initiation (or return to) dialysis, or another transplant }\end{array}$ \\
$\begin{array}{ll}\text { Infectious risk } \\
\text { Neoplastic risk }\end{array}$ & $\begin{array}{l}\text { The risk of contracting infectious disease through the transplanted organ } \\
\text { The risk of contracting a tumour through the transplanted organ }\end{array}$ \\
\hline
\end{tabular}

transplant centre can have a maximum of 250 patients enrolled in its waiting list. There is no age limit for kidney transplant eligibility. All transplant activities in Italy are coordinated by the 'Centro Nazionale Trapianti' (Transplant National Centre) and three multi-region coordination programs - Nord Italia Transplant program (NITp), Associazione Interregionale Trapianti (AIRT), Organizzazione Centro-Sud Trapianti (OCST) - that cover the entire territory. The Pancreas and Kidney Transplant Unit of the University of Padova belongs to the NITp, which coordinates the transplant activities in five Italian regions in the north of the country. The allocation scheme for kidney transplants in these regions is managed by the NITp, which is responsible for the assignments of available organs from deceased donors to the single transplant centre. ${ }^{4}$

We administered a survey consisting of a set of questions on socio-economic characteristics and 16 questions that constituted a DCE to all the 250 patients on the waiting list for a kidney transplant at the Kidney and Pancreas Transplantation Unit of the School of Medicine, University of Padova. ${ }^{5}$ A psychologist conducted face-to-face interviews using a Paper Assisted Personal Interview (PAPI) methodology. The interviewer explained the experiment and obtained informed consent from each participant. Two participants were discarded due to their psychological condition. The remaining 248 patients completed the questionnaire. Interviews took place on the day in which patients visited the transplant centre for their routine annual check-up. ${ }^{6}$ Ethical approval for the study was obtained from the Ethical Committee of the University of Padova.

\section{Design of the experiment}

Discrete choice experiments (DCEs) are used to elicit individuals' stated preference parameters among alternative medical treatments (de Bekker-Grob et al., 2012; Ryan and Gerard, 2003; Lancsar et al., 2011; Meenakshi et al., 2012; Fischer et al., 2018). Treatments are described by their underlying attributes, consistent with the Lan-

\footnotetext{
4 Patients with end-stage renal disease can also receive an organ from a living compatible donor. Typically, this living donor is a relative of the patient. Patients who have an incompatible willing donor can also participate in Kidney Paired Exchange programs, which are designed to increase the number of transplants from living donors by exchanging donors among incompatible pairs. In this paper, we do not mention the option of living donations because none of the patients involved in our study had a compatible or incompatible living donor. For further information visit www.trapianti.sanita.it.

5 A copy of the survey instrument can be found in the appendix.

6 The first interviews took place on April 14, 2015; the last took place on June 6, 2017.
}

casterian theory of demand (Lancaster, 1966), and the alternatives are formed by varying the values taken by a set of attributes. Typically, each individual is asked to choose their preferred alternative from a list of choice sets, thus contributing multiple observations (Lancsar et al., 2017). The opportunity to include continuous variables, such as cost or waiting time attributes, allows researchers to estimate willingness to pay (WTP) (Hole, 2008; Nieboer et al., 2010) or willingness to wait (WTW) (Brown et al., 2015; Rousseau and Rousseau, 2012; Hagemi et al., 2017; Marshall et al., 2018) for variations in attributes' levels. Those measures constitute meaningful preference parameters if the results of the DCE are interpreted within a random utility framework (McFadden, 1974; McFadden and Train, 2000).

We determined the attributes and levels in consultation with surgeons from the same transplant centre as the patients. Qualitative methods are increasingly used to determine attributes and levels in the design of DCE (Coast and Horrocks, 2007); the consultation allowed us to design the DCE with exactly the same wording the doctors would use when discussing alternative treatments with patients. As an example, medical doctors describe the infectious and neoplastic risks of a kidney to patients as either standard or augmented. This is an explicit choice made to emphasise to patients that a zero-risk kidney does not exist. Attributes and levels are reported in Table 1.

Two attributes are enumerable (i.e., waiting time and expected graft survival). Waiting time is the number of months that patients can expect to wait to undergo the proposed transplant. This is our "numeraire", i.e., the attribute that allows us to compute WTW for changes in other attributes. The expected graft survival is the expected number of years of functioning of the transplanted organ. In the case of organ failure, patients return to dialysis and can be re-transplanted.

Infectious and neoplastic risk are qualitative attributes, but the levels are ordinal: augmented risk is higher than standard. A standard-risk kidney is an organ for which the evaluation process did not identify any risk factors for transmittable disease. Standard risk is the most frequent condition in the assessment of donors and grafts. Doctors speak of standard-risk, and not zero-risk kidneys since infectious or neoplastic diseases can be transmitted even if guidelines and good clinical practices are followed. An organ is labelled as an augmented risk if certain medical tests could not be performed or the donor was engaged in certain risky behaviours prior to death (e.g., use of drugs) that increase the probability of infections that cannot be detected immediately after contraction (Venettoni et al., 2006). 


\section{Table 2}

Illustration of a choice task (original in Italian): which of the two treatments would you prefer? Put an X below the chosen treatment.

\begin{tabular}{lll}
\hline & Treatment A & Treatment B \\
\hline Waiting time & 6 months & 6 months \\
Expected graft Survival & 20 years & 15 years \\
Infectious risk & Standard & Standard \\
Neoplastic risk & Augmented & Standard \\
Your choice? & $\square$ & $\square$ \\
\hline
\end{tabular}

A full factorial design using the attributes and levels hitherto-defined would have resulted in 48 possible profiles $\left(4 * 3 * 2^{2}\right)$, leading to 1128 possible choice sets, which are clearly too many to be implemented in a DCE. McFadden and Train (2000) demonstrated that completeness, monotonicity and transitivity of preferences are necessary conditions to interpret parameters' estimates obtained from a DCE as preference parameters. Therefore, we restricted the design to 16 choice sets using a D-efficient algorithm that searches for a list of choice sets in which dominant alternatives do not appear, choice sets are not repeated, and the number of choice sets for which the answer can be inferred from the previous one is minimised (assuming transitivity and monotonicity). ${ }^{7}$ The number of choice sets to be included in our experiment was determined by a pilot study conducted by taking students as subjects, wherein we found no evidence of any fatigue effect with 16 choice sets. To avoid the complexity of the design, we used only main-effects design, and we did not allow the estimation of interaction effects between attributes at the design stage. After consultation with surgeons this seemed a reasonable specification given the different aetiology of tumours and infectious diseases transmittable with a transplant. ${ }^{8}$

Table 2 reports an example of a choice set. Patients were asked which of the two alternatives (A or B) they would prefer in each choice set. The four attributes taking specific levels described each alternative.

Like a few other studies (Rigby et al., 2010; Hasund et al., 2011; Milte et al., 2018; Patterson et al., 2019), we did not include an opt-out option. We asked patients which treatment they would prefer between the two proposed alternatives, rather than which one they would have chosen, as a patient could prefer to remain on the waiting list rather than receiving either of the two organs. We did not include the opt-out option as "leaving the transplant program" or "remaining on the waiting list" in the choice sets. A crucial feature of the NITp allocation protocol is that there is no penalty for a patient who declines to undergo a kidney transplant. If the opt-out option had been stated as "leaving the transplant program", the experiment could have provoked distress because patients could

\footnotetext{
7 When a full factorial design is not feasible, the most common metric in design construction is D-optimality (Johnson et al., 2013). We then modified the AlgDesign Package in R (Aizaki and Nishimura, 2008) to be theory-consistent as explained.

8 This assumption does not imply attributes are independent. As explained in the next section, at the estimation stage we did not impose independence and estimated the parameters allowing for full correlation amongst them.
}

interpret this option as a threat. ${ }^{9}$ Moreover, "leaving the transplant program" is a dominated strategy for patients, precisely because patients keep their priority on the waiting list when they decline a transplant. As explained above, we did not include dominated alternatives in the choice sets. ${ }^{10}$ If the opt-out option had been stated as "remaining on the waiting list", then it would have been inaccurately described because this choice corresponds to an expected treatment that depends on the beliefs of the patients. In other words, if a patient prefers to remain on the waiting list, this means she believes that in the future a kidney will be offered that is preferable to both the proposed treatments. The opt-out option would be a third potential treatment comparable ex-ante to those offered, but described as "remaining in the waiting list" rather than with attribute levels.

\section{Econometric analysis}

Response data from DCEs are modelled within a random utility maximisation framework (McFadden, 1974). The utility obtained by patient $m$ from choosing kidney transplant alternative $t$ in a choice set $s$ is specified as a function

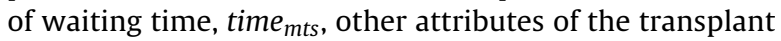
(namely graft survival and infectious and neoplastic risk) included in the vector $\boldsymbol{x}_{m t s}$, an alternative specific constant (ASC), and a random term, $\varepsilon_{m t s}$, Extreme Value distributed with variance $\mu_{m}^{2}\left(\pi^{2} / 6\right)$.

$$
\begin{aligned}
U_{m t s} & =V\left(\text { time }_{m t s}, \boldsymbol{x}_{m t s}, A S C\right)+\varepsilon_{m t s} \\
& =-\alpha_{m} \text { time }_{m t s}+\boldsymbol{\beta}_{m}^{\prime} \boldsymbol{x}_{m t s}+A S C+\varepsilon_{m t s}
\end{aligned}
$$

$A S C_{t}$ controls for the 'residual' mean influence of unobservable sources of marginal utility (Berry et al., 1995). Since in the DCE at hand alternatives are randomly assigned label A or B in each choice set, this term controls for left-to-right (reading) bias (Ryan et al., 2018).

The probability patient $m$ chooses treatment $A$ in choice set $s$ is defined as

$$
\begin{aligned}
& P_{m A s}=\operatorname{Prob}\left(U_{m A s}-U_{m B s}>0\right)=1-\operatorname{Prob}\left(U_{m A s}-U_{m B s} \leq 0\right) \\
& 1-\operatorname{Prob}\left(\varepsilon_{m a A s}-\varepsilon_{m B s} \leq V\left(\text { time }_{m A s}, \boldsymbol{x}_{m A s}, A S C\right)\right. \\
& \left.-V\left(\text { time }_{m B s}, \boldsymbol{x}_{m B s}, A S C\right)\right)
\end{aligned}
$$

If patients' preferences are complete, monotone, and transitive, assuming a distribution for the taste coefficients $\alpha_{m}$ and $\boldsymbol{\beta}_{m}, P_{m A s}$ defines a latent variable model that can be estimated with a mixed multinomial logit (McFadden and Train, 2000).

The coefficients $\alpha_{m}$ and $\boldsymbol{\beta}_{m}$ represent the preferences of patient $m$. Alternatively, an easier way to interpret heterogeneity in preferences is to resort to Willingness to Wait

\footnotetext{
9 It is important to keep in mind that our experiment was run with "real" patients waiting for a transplant. We took great care to avoid any question which might cause confusion about the rules of the current allocation protocol.

10 Patients can cancel their registration from the waiting list at any time. Therefore, we know that for each patient, remaining on the waiting list is preferred to leaving it.
} 
(WTW). The WTW for attribute $k$ is the number of months patient $m$ is willing to wait for an extra level of attribute $k$, that is, the marginal rate of substitution between attribute $k$ and time:

$W T W_{k m}=-\frac{\partial U / \partial x_{k, m}}{\partial U / \partial \text { time }_{m}}=-\frac{\beta_{k, m}}{\alpha_{m}}$

The distributional assumptions on the preference coefficients determine the distribution of $W T W_{k m}$. The standard approach to ensuring a well-defined distribution for $W T W_{k m}$ is to assume that the coefficient $\alpha_{m}$ is not random, implying each vector $\boldsymbol{W} \boldsymbol{T} \boldsymbol{W}_{m}$ has the same distribution of $\boldsymbol{\beta}_{m}$. This approach is problematic since $\varepsilon_{m t s}$ variance depends on $\mu_{m}$, a patient-specific scale-parameter. If $\alpha_{m}$ is not random, then for all $k, W T W_{k m}$ are not scale free, and variation in $\mu_{m}$ can induce variation in $W T W_{k m}$, holding taste coefficients constant. In other words, variation in scale will be confounded with the variation in WTW for transplant attributes (Train and Weeks, 2005). An alternative approach is to assume $\alpha_{m}$ to be log-normally distributed. Still, this would result in unrealistic estimates of the mean and standard deviation of WTW values and heavily skewed distributions (Hole and Kolstad, 2012). To overcome these problems, we follow Hensher and Greene (2011), re-parametrise the model, and estimate the multinomial mixed logit in WTW space. The individual utility function (1) can be rewritten as follows:

$$
\begin{aligned}
U_{m t s} & =-\alpha_{m}\left[\text { time }_{m t s}-\left(\frac{1}{\alpha_{m}}\right) \boldsymbol{\beta}_{m}^{\prime} \boldsymbol{x}_{m t s}\right]+A S C+\varepsilon_{m t s} \\
& =-\alpha_{m}\left[\text { time }_{m t s}-\boldsymbol{W T W}_{m}^{\prime} \boldsymbol{x}_{m t s}\right]+A S C+\varepsilon_{m t s}
\end{aligned}
$$

The time attribute parameter $\alpha_{m}$ becomes the normalising constant in the WTW space representation. We can now directly assume a distribution of $\mathbf{W} \boldsymbol{T} \boldsymbol{W}_{m}$ rather than of the original preference coefficients. We assume each $W T W_{k m}$ to be normally distributed and, following Hole and Kolstad (2012), $\alpha_{m}$ to be log-normally distributed. Moreover, we allow the random parameters to correlate each other (i.e., we do not restrict the Variance Covariance matrix of the estimated parameters to be diagonal). The model hitherto-outlined allows for heterogeneity in unobservable characteristics. Nevertheless, preferences in kidney transplantation may also differ along observable dimensions (Roth and Sönmez, Ünver, 2004). Whether or not $\boldsymbol{W T} \boldsymbol{W}_{m}$ correlates with patients' observable characteristics constitutes an important question from a policy perspective: observable characteristics can easily be included in kidney allocation protocols.

We focus on age and duration of dialysis. Age has been found to affect time and risk preferences in many domains (Morin and Suarez, 1983; Bishai, 2004), while patients who have spent longer periods in dialysis are typically given priority in allocation protocols.

Fig. 1 presents the kernel density plots of the distributions of the duration of dialysis and age, respectively. The duration of dialysis is left-skewed, with a larger part of the mass between zero and five years but a long right tail (Fig. 1a) accounting for patients for whom it is more difficult to find a compatible kidney. Conversely, the age distribution is fairly symmetric (Fig. 1b). The difference in the skewness of the two distributions, and therefore the low correlation between age and duration of dialysis, can be explained by the fact that the probability of a patient finding a compatible organ depends primarily on the tissue type, regardless of age.

To account for age and duration of dialysis, we extend the model allowing for mean heterogeneity in WTW-space, i.e., we allow parameter heterogeneity to be partly systematic in terms of observed variables (see Sarrias et al., 2016). The mean heterogeneity can be written as:

$\mathrm{WTW}_{\mathrm{k}, \mathrm{m}}=\boldsymbol{W} \boldsymbol{T} \boldsymbol{W}+\boldsymbol{\Delta} \mathrm{z}_{\mathrm{i}}+\mathbf{L} \boldsymbol{\rho}_{\mathrm{i}}$,

where $\boldsymbol{\Delta}$ is a matrix of parameters, $z_{i}$ is a vector of covariates (in our case it is a $2 \times 1$ vector containing age and duration of dialysis) that do not vary across choice tasks, and $\boldsymbol{\rho} \sim N(\mathbf{0}, \mathrm{I})$.

The conditional mean vector varies across patients through $\mathrm{z}_{\mathrm{i}}$ :

$\mathbf{E}\left(\mathrm{WTW}_{\mathrm{k}, \mathrm{m}} \mid \mathrm{z}_{\mathrm{i}}\right)=\mathbf{W T W}+\Delta \mathrm{z}_{\mathrm{i}}$

Detailed discussion on how to account heterogeneity around the mean of the distribution in the mixed logit framework can be found in Greene et al. (2006) and Bhat (2000). The coefficient of the 'waiting time' attribute is still log-normally distributed with mean $\bar{\alpha}$ and standard deviation $\sigma_{\alpha}$, but now the mean is a function of the covariates:

$\alpha_{m}=\exp \left(\bar{\alpha}+\delta_{1}\right.$ age $+\delta_{2}$ duration of dialysis $\left.+\sigma_{\alpha} \eta_{m}^{k}\right)$

$\eta_{m}^{k} \sim N(0,1)$

The WTW for the $k$ th attribute is normally distributed with mean $\overline{W T W_{k}}$ and standard deviation $\sigma_{k}$ but now the mean is a function of the covariates:

$$
\begin{aligned}
& W T W_{m k}=\overline{W T W_{k}}+\delta_{1 k} \text { age }+\delta_{2 k} \text { duration of dialysis } \\
& +\sigma_{k} \eta_{m}^{k}
\end{aligned}
$$

$\eta_{m}^{k} \sim N(0,1)$

The above formulation implies that, for instance, the marginal effect on the WTW for graft survival varies by age and duration of dialysis. In other words, we allow $W T W_{m k}$ to vary across individuals both randomly and systematically with age and duration of dialysis.

\section{Results}

Fig. 2 reports the kernel density plots of the WTW estimates. We estimated the models using the maximum simulated likelihood method with 10,000 scrambled Sobol draws (Czajkowski and Budziński, 2019). We used zeros as starting values to estimate a basic multinomial logit in WTW-space model. These preliminary estimates were then used as starting values for estimating a mixed logit in WTW-space model with normal distribution for all the 


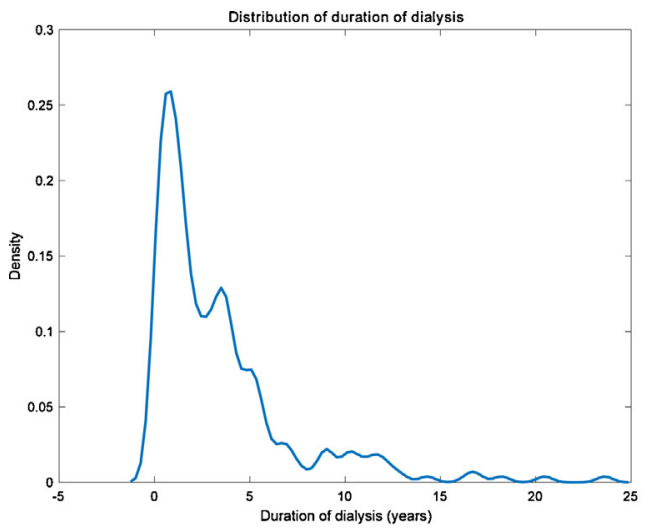

(a) Duration of dialysis

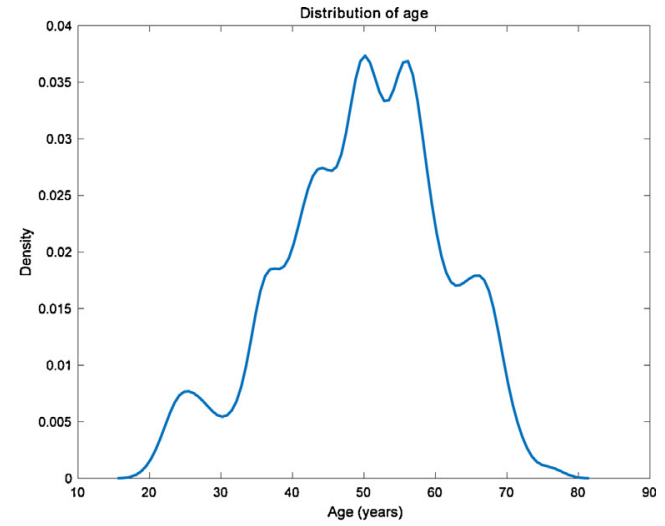

(b) Age

Fig. 1. Kernel plots of the distribution of covariates (duration of dialysis and age).

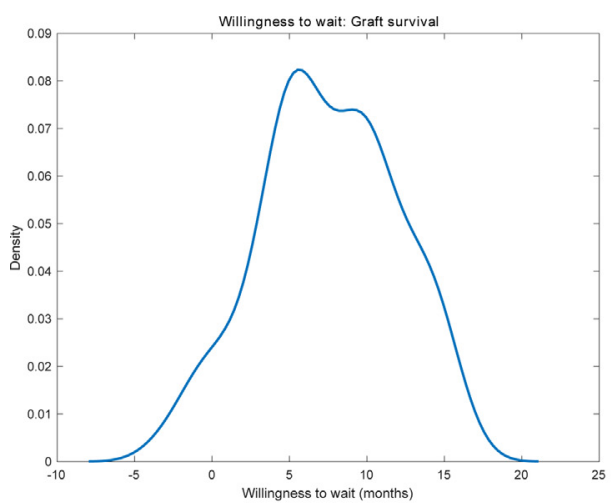

(a) WTW for extra year of survival

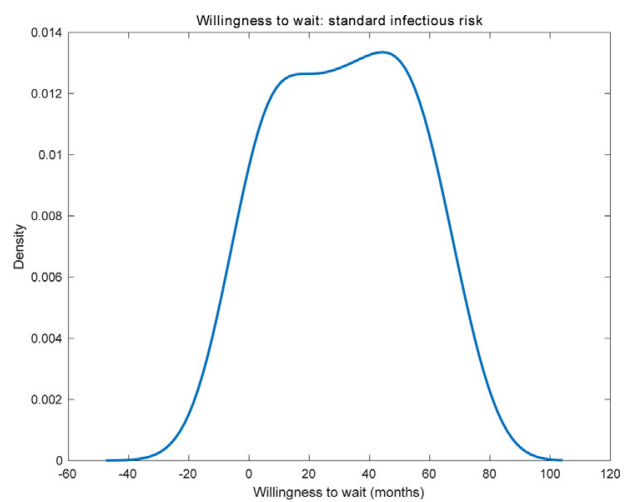

(b) WTW for standard infectious risk

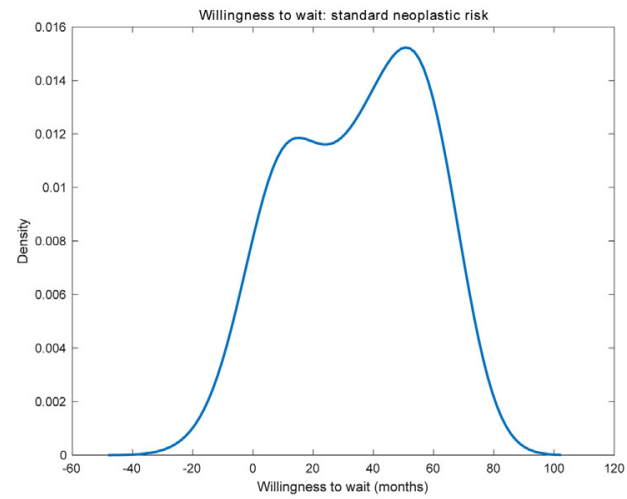

(c) WTW for standard neoplastic risk

Fig. 2. Kernel density plots of the distribution of individual WTW.

attributes, which in turn were used to estimate the main model. ${ }^{11}$

11 We also estimated the models using alternative starting values, obtaining consistent results.
The plots immediately highlight a substantial dispersion in the distributions, pointing to significant preference heterogeneity across patients.

The WTW for an extra year of graft survival, presented in panel $2 \mathrm{a}$, is concentrated at about seven months. In 
Table 3

Baseline multinomial mixed logit, empirical distributions first and second moment.

\begin{tabular}{|c|c|c|}
\hline & $\begin{array}{l}(1) \\
\text { (Mean) }\end{array}$ & $\begin{array}{l}(2) \\
(\mathrm{SD})\end{array}$ \\
\hline Waiting time $(\alpha)$ & $\begin{array}{l}-2.673^{* * *} \\
(0.078)\end{array}$ & $\begin{array}{l}0.868^{* * *} \\
(0.116)\end{array}$ \\
\hline$W_{T} W_{\text {survival }}$ & $\begin{array}{l}7.516^{* * *} \\
(0.509)\end{array}$ & $\begin{array}{l}5.421^{* * *} \\
(0.497)\end{array}$ \\
\hline$W_{\text {standard infectious risk }}$ & $\begin{array}{l}31.462^{* * *} \\
(2.173)\end{array}$ & $\begin{array}{l}26.815^{\text {*** }} \\
(2.733)\end{array}$ \\
\hline$W_{\text {standard neoplastic risk }}$ & $\begin{array}{l}34.594^{* * *} \\
(2.400)\end{array}$ & $\begin{array}{l}26.541^{\text {*** }} \\
(3.175)\end{array}$ \\
\hline ASC & $\begin{array}{l}3.069^{* * *} \\
(0.604)\end{array}$ & - \\
\hline \multicolumn{3}{|l|}{ Model diagnostics } \\
\hline Log-likelihood (LL) at convergence & -2039.912 & \\
\hline McFadden's pseudo- $R^{2}$ & 0.229 & \\
\hline Akaike Information Criterion (AIC) $/ n$ & 1.1079 & \\
\hline Bayesian Information Criterion (BIC) $/ n$ & 1.1096 & \\
\hline$n$ (observations) & 3818 & \\
\hline$r$ (respondents) & 248 & \\
\hline
\end{tabular}

Standard errors in parentheses. ${ }^{*} p<0.1,{ }^{* *} p<0.05,{ }^{* * *} p<0.01$.

Abbreviations: ASC: Alternative specific constant, SD: standard deviation.

Fig. 2b and c, the distributions are bimodal, suggesting even more heterogeneity in WTW for changes in the risk attributes. The left tails of the distributions are in the negative domain, implying that some respondents prefer shorter graft survival (about 5\%) or higher infections (about $8 \%$ ) and neoplastic risk (2\%). This is because we assumed $\mathrm{WTW}_{\mathrm{m}}$ to be normally distributed, i.e., we did not impose any restriction on the sign of WTW estimates. To test for sensitivity to the chosen distribution, we estimated the individual coefficients assuming that all parameters are log-normally distributed, thus imposing a lower bound at zero. Results reported in Appendix A.4, are consistent across models in terms of signs and significance. However, as in Hole and Kolstad (2012), fitting the parameters to a log-normal distribution resulted in thicker right-hand tails with unreasonable WTW values.

\subsection{Baseline results}

In Table 3, we report the first and second moments of the empirical distribution of each parameter estimate. Correlations between parameters estimates are reported in Appendix A.1. The mean values are all significantly different from zero and have the expected signs. The standard deviations (SD, column 2) are also significant and sizeable, supporting the evidence in favour of preference heterogeneity.

The mean WTW for a kidney that will offer an additional year of graft survival is about seven months and a half. ${ }^{12}$ On average, patients are willing to wait, ceteris paribus, 31-35 months longer for a kidney of standard risk as compared to one of augmented risk. The mean ASC is statistically significant, indicating the presence of left-to-right biases in our

\footnotetext{
12 We run an alternative version of the model to estimate WTW for a 5 -year graft survival differences, the same time span as in the proposed levels of the graft survival attributes. Results are reported in Appendix A.1 and are in line with those reported here.
}

data, a result common to many other DCEs in the health domain (see, e.g., Determann et al., 2017).

Finally, we document the sensitivity of our results to modelling choices in line with Hole and Kolstad (2012), i.e., we estimated a standard multinomial logit model, and a mixed logit model restricting the random parameter estimates to be uncorrelated. Moreover, we repeated the full set of estimates in the preference space rather than in the WTW space. Results are reported in Appendix A.3, along with plots comparing the distributions of individual WTW estimates. Models in the preference space fit the data better than models in the WTW space. However, the distributions of WTW that are derived from models in the preference space exhibit a large variance, which translates into the unreasonable implication that many patients are willing to wait an enormous length of time to have an attribute. Our results are consistent with Hensher (2006) and Sonnier et al. (2007), besides Hole and Kolstad, 2012.

Estimates in Table 3 do not account for systematic differences driven by observable characteristics. As explained in Section 4, there are good reasons to investigate whether at least part of the heterogeneity can be associated with differences in age and duration of dialysis. We now discuss the estimates of the model accounting for mean heterogeneity reported in Table 4.

\subsection{Age}

Time and risk preferences have been found to vary with age in several domains. We expect that differences in life expectancy cause WTW in attributes of kidney transplantation to vary according to patients' age. Column 3 of Table 4 shows the interaction terms, which allow us to examine the deterministic heterogeneity around the means of the estimated WTW parameters. For example, the interaction term between age and graft survival is used to test if older patients will have a lower WTW for a kidney that will offer an extra year of graft survival than younger patients. All the interaction coefficients are statistically significant at the conventional level. All else equal, every additional year of age reduces WTW for an extra year of graft survival by 0.1 months (3 days). Similarly, an extra year of age reduces the WTW for a standard infectious risk by 0.3 months ( 9 days) while the interaction between age and standard neoplastic risk failed to reach significance.

Observable differences in age account for a significant proportion of heterogeneity in WTW: Table 5 shows the WTW for each attribute at different values of age using the coefficient estimates from Table 4 of Eqs. (7) and (8). In each line of the table, we assign specific values for age (20-90) and fix the duration of dialysis at its mean (3.4 years). A patient with an age of 20 years and with 3.4 years of dialysis is willing to wait 10.5 months for each expected additional year of functioning. WTW drops substantially with age: a 75 years old patient with the same duration of dialysis ( 3.4 years) is willing to wait only five months for each additional year of graft survival.

Our findings complement previous studies that have used administrative data. For instance, using data from the Scientific Registry of Transplant Recipient database and based on survival models, Schold and Meier-Kriesche 
Table 4

Mean heterogeneity in WTW-space model results.

\begin{tabular}{|c|c|c|c|c|}
\hline & $\begin{array}{l}(1) \\
\text { (Mean) }\end{array}$ & $\begin{array}{l}(2) \\
(\mathrm{SD})\end{array}$ & $\begin{array}{l}(3) \\
\text { (Age) }\end{array}$ & $\begin{array}{l}\text { (4) } \\
\text { (Duration of dialysis) }\end{array}$ \\
\hline Waiting time $(\alpha)$ & $\begin{array}{l}-3.437^{* * *} \\
(0.362)\end{array}$ & $\begin{array}{l}0.873^{* * *} \\
(0.134)\end{array}$ & $\begin{array}{l}0.017^{* *} \\
(0.007)\end{array}$ & $\begin{array}{l}-0.018 \\
(0.022)\end{array}$ \\
\hline$W_{\text {Turvival }}$ & $\begin{array}{l}11.8197^{* * * *} \\
(2.302)\end{array}$ & $\begin{array}{l}5.258^{* * *} \\
(0.496)\end{array}$ & $\begin{array}{l}-0.101^{* *} \\
(0.042)\end{array}$ & $\begin{array}{l}0.228^{* *} \\
(0.101)\end{array}$ \\
\hline$W_{\text {Ttandard infectiousrisk }}$ & $\begin{array}{l}45.309^{* * *} \\
(9.545)\end{array}$ & $\begin{array}{l}26.958^{* * *} \\
(2.742)\end{array}$ & $\begin{array}{l}-0.320^{*} \\
(0.187)\end{array}$ & $\begin{array}{l}0.624 \\
(0.623)\end{array}$ \\
\hline$W_{T W}$ standard neoplasticrisk & $\begin{array}{l}46.518^{* * *} \\
(10.549)\end{array}$ & $\begin{array}{l}26.689^{* * *} \\
(3.056)\end{array}$ & $\begin{array}{l}-0.274 \\
(0.199)\end{array}$ & $\begin{array}{l}0.576 \\
(0.561)\end{array}$ \\
\hline ASC & $\begin{array}{l}6.238 * * \\
(2.782)\end{array}$ & & $\begin{array}{l}-0.056 \\
(0.054)\end{array}$ & $\begin{array}{l}-0.077 \\
(0.222)\end{array}$ \\
\hline \multicolumn{5}{|l|}{ Model diagnostics } \\
\hline Log-likelihood (LL) at convergence & -2032.378 & & & \\
\hline McFadden's pseudo- $R^{2}$ & 0.232 & & & \\
\hline Akaike Information Criterion (AIC) $/ n$ & 1.0798 & & & \\
\hline Bayesian Information Criterion (BIC) $/ n$ & 1.1273 & & & \\
\hline$n$ (observations) & 3818 & & & \\
\hline$r$ (respondents) & 248 & & & \\
\hline
\end{tabular}

Standard errors in parentheses. ${ }^{*} p<0.1,{ }^{* *} p<0.05,{ }^{* * *} p<0.01$.

Abbreviations: ASC: alternative specific constant, SD: standard deviation. Standard errors in parentheses.

Table 5

WTW for different age levels and for an average duration of dialysis (3.4 years).

\begin{tabular}{llll}
\hline Age (years) & $(1)$ & $(2)$ & $(3)$ \\
& WTW $_{\text {survival }}$ & WTW $_{\text {infectious }}$ & WTW $_{\text {neoplastic }}$ \\
\hline 20 & 10.57 & 41.03 & 43.00 \\
25 & 10.07 & 39.43 & 41.63 \\
30 & 9.56 & 37.83 & 40.26 \\
35 & 9.06 & 36.23 & 38.89 \\
40 & 8.55 & 34.63 & 37.52 \\
45 & 8.05 & 33.03 & 36.15 \\
50 & 7.54 & 31.43 & 34.78 \\
55 & 7.04 & 29.83 & 33.41 \\
60 & 6.53 & 28.23 & 32.04 \\
65 & 6.03 & 26.63 & 30.67 \\
70 & 5.52 & 25.03 & 29.30 \\
75 & 5.02 & 23.43 & 27.93 \\
80 & 4.51 & 21.83 & 26.56 \\
85 & 4.01 & 20.23 & 25.19 \\
90 & 3.50 & 18.63 & 23.82 \\
\hline
\end{tabular}

The WTW values are obtained using Equation (8) and the coefficient estimates from Table 4, i.e., we assigned specific values for age (20-90) and take the mean of duration of dialysis ( 3.4 years).

(2006) showed that older patients (those 65 years and above) had longer life expectancy when they accepted an ECD after two years of dialysis ( 5.6 years) compared with waiting for a standard kidney ( 5.3 years) or a living donation ( 5.5 years) after four years of dialysis. The same study also indicated that younger patients (18-39 years old) had longer life expectancy with a living donation (27.6 years) or standard kidney (26.4 years) after four years on dialysis compared with an ECD after two years of dialysis (17.6 years). In the study by Jay et al. (2017), pre-emptive transplantation of "non-ideal" kidneys on recipients over the age of 60 reduces mortality hazard compared with the waitlist, including transplant recipients of standard quality kidneys. Therefore in our experiment, the estimated preference of the patients are also consistent with a simple model of life expectancy maximisation: patients are willing to accept "worse" kidneys as they age, and previous literature shows that this choice would increase their life expectancy.

We provide evidence that younger patients are willing to wait longer for a kidney transplant characterised by better levels of the attributes (i.e., an extra year of graft survival and standard infectious risk) compared to older patients. In Appendix B.1, we report evidence that the whole distribution is shifted to the left for older individuals, suggesting that keeping patients on the waiting list as they age, may change their preferences. However, accounting for the dynamics in preferences and WTW as age increases would necessitate observing a patient at different points in time, which is beyond the scope of this study.

\subsection{Duration of dialysis}

Patients who are diagnosed with irreversible chronic kidney failure and lack access to pre-emptive transplantation need to undergo dialysis treatment whilst waiting for kidney transplantation. The length of stay on dialysis depends, among other factors, on initial health condition and on the probability of finding a compatible organ, which depends not only on their blood type but also, even more importantly, on their tissue type. Every individual has some donor-specific anti-HLA (Human Leukocyte Antigen) proteins that prevent the patient from receiving a kidney from certain donors. Roughly speaking, the more of these antibodies a patient has, the less likely the patient is to find a compatible organ because the patient has to wait for an HLA-compatible kidney. This means that transplant candidates with longer dialysis history are often highly sensitised patients with a large number of HLA proteins. As a consequence, almost all allocation mechanisms prioritise individuals that have spent a long period of time on dialysis for reasons of fairness. This allocation rule may not be optimal, however, if preferences change with the duration of dialysis. This is precisely what we want to investigate in this section: how $\mathbf{W T} \boldsymbol{W}_{m}$ differs according to the duration of dialysis. 
Table 6

WTW for different duration of dialysis and for an average age (50 years).

\begin{tabular}{|c|c|c|c|}
\hline \multirow{2}{*}{$\begin{array}{l}\text { Duration of } \\
\text { dialysis (years) }\end{array}$} & (1) & $(2)$ & (3) \\
\hline & $W_{\text {Survival }}$ & $W_{T} W_{\text {infectious }}$ & $W_{T} W_{\text {neoplastic }}$ \\
\hline 0.5 & 6.88 & 29.62 & 33.11 \\
\hline 1 & 7.00 & 29.93 & 33.39 \\
\hline 2 & 7.23 & 30.56 & 33.97 \\
\hline 3 & 7.45 & 31.18 & 34.55 \\
\hline 4 & 7.68 & 31.81 & 35.12 \\
\hline 5 & 7.91 & 32.43 & 35.70 \\
\hline 6 & 8.14 & 33.05 & 36.27 \\
\hline 7 & 8.37 & 33.68 & 36.85 \\
\hline 8 & 8.59 & 34.30 & 37.43 \\
\hline 9 & 8.82 & 34.93 & 38.00 \\
\hline 10 & 9.05 & 35.55 & 38.58 \\
\hline 11 & 9.28 & 36.17 & 39.15 \\
\hline 12 & 9.51 & 36.80 & 39.73 \\
\hline 15 & 10.19 & 38.67 & 41.46 \\
\hline 20 & 11.33 & 41.79 & 44.34 \\
\hline
\end{tabular}

The WTW values are obtained using Eq. (8) and the coefficient estimates from Table 4, i.e., we assign specific values for the duration of dialysis (0.5-20) and keeping age at its mean value (50 years).

Results are presented in column 4 of Table 4 . The coefficients of 'duration of dialysis' is positive and statistically significant for graft survival, while significance is lost for the risk attributes. This means that the longer the duration of dialysis, the longer patients are willing to wait for a kidney with a better-expected graft survival. ${ }^{13}$ To be more specific, comparing two patients A and B who only differ because patient $A$ spent one more year on dialysis than $B$, on average patient $A$ is willing to wait 0.22 months (approximately a week) more than B for a kidney that will offer an extra year of graft survival.

In Table 6, we show how WTW for changes in each kidney transplantation attribute varies for different values of duration of dialysis, holding age fixed at its mean value (50 years). The model indicates that for a patient with an average age of 50 years and dialysis duration of one year, the WTW for a kidney that will offer one additional year of graft survival is about seven months. A 50-year old patient with six years on dialysis is willing to wait more than eight months for a kidney that will provide an additional year of graft survival. As we did for age, we report in Appendix B.2 some further analysis on how the shape of the distribution of WTW changes with the duration of dialysis. We show that the distribution is shifted to the right, and the shape changes substantially for patients with longer duration of dialysis. In other words, the degree of preference heterogeneity increases with the duration of dialysis.

\section{A simulation exercise}

The substantial preference heterogeneity among patients waiting for a transplant suggests that it is possible to increase their welfare by including patients' preferences in the design of kidney allocation protocols. We ran a simulation exercise to give a sense of how much the potential outcomes of interest would change if a

\footnotetext{
13 The duration of dialysis was obtained by taking the difference between the date of interview and the starting date of dialysis.
}

preference-based allocation protocol was adopted. To do so, we compared a stylised protocol that captures the salient features of the existing protocol at the Kidney and Pancreas Transplantation Unit of the University of Padova, with a second stylised protocol that aims to maximise the unweighted sum of patients' utilities.

The first step in the simulation process is to simulate a stream of donors kidneys. We extracted with reinsertion a kidney from the records of a database that included the last one hundred kidneys offered to patients in Padova in 2019 from the NITp organ procurement database. We retrieved all the information needed to compute the expected graft survival, infectious and neoplastic risk. We derived the expected graft survival of a kidney from its Kidney Donor Risk Index (KDRI) (Rao et al., 2009). The KDRI expresses the relative risk of graft failure for a given donor compared to a median donor, and it is based on ten clinical and demographic donors' characteristics. ${ }^{14}$ We computed the index using the online KDRI calculator available on the U.S. Department of Health \& Human Services website, and mapped it into years of graft survival following the guidelines of the Organ Procurement and Transplantation Network (2019). Regarding neoplastic and infectious risk, none of the kidneys included in the database were classifiable as an augmented risk. We then assigned the augmented risk label to $5 \%$ of the kidneys in the database chosen randomly. ${ }^{15}$

In the second step of the simulation, we determine the compatible patients in the pool of potential transplant candidates. To do so, we use the panel-reactive antibody (PRA) score of each patient to determine the probability of compatibility. The PRA test is a standard immunological test performed on patients awaiting a transplant and measures the degree of alloimmunity in a graft recipient, therefore quantifying the risk of graft rejection. The PRA score represents the proportion of the kidney population to which the person tested would not be compatible. In the second step, we draw from the pool of remaining patients those compatible based on their PRA scores.

In the third step of the simulation, compatible patients are ranked according to the benchmark and the preferencebased protocols. Since the 90 s, one of the most important criteria to establish priority in the waitlist has been the waiting time, which most of the time corresponds to the duration of dialysis (Sirchia et al., 1998). Our benchmark protocol then ranks compatible patients based on the duration of dialysis at the time of arrival of the donor's kidney. Conversely, in the preference-based protocol, we first compute the expected utility from the transplant. This is based on Eq. (4) using the estimated coefficients summarised in Fig. 2, kidney characteristics (expected graft survival, and infectious and neoplastic risks), and the time since the

\footnotetext{
14 The KDRI is computed with respect to a reference to the American donors' population, which may differ from the Italian one. Still, it is common practice to use it to estimate the expected survival of the Italian transplanted patients (Gandolfini et al., 2014).

15 We experimented with bigger and smaller shares of reassignment, as well as with no reassignment. Results are by and large the same as reported in the paper. Note that all the offered kidneys were transplanted, pointing to the average high quality of the kidneys.
} 


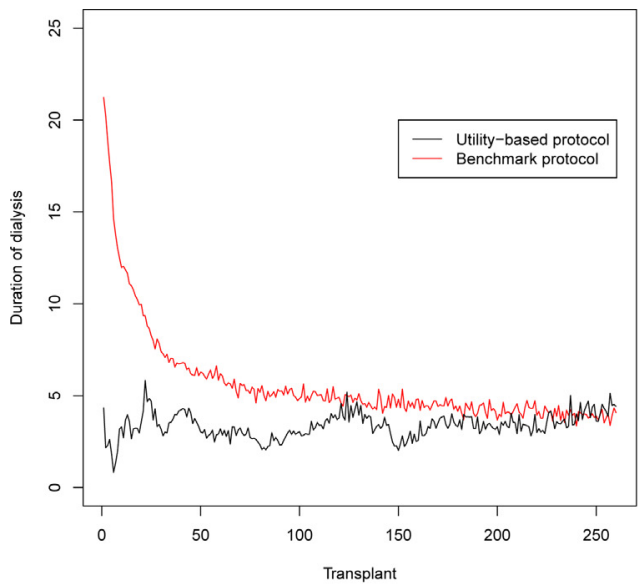

(a) Duration of dialysis at transplant

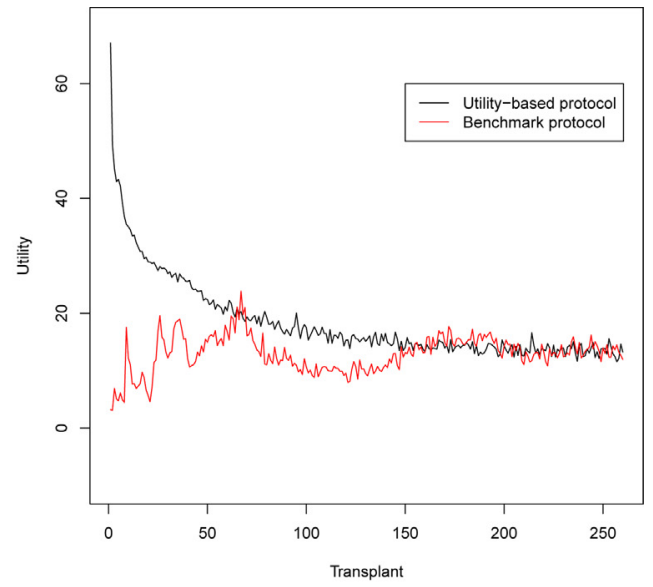

(b) Utility of transplanted patients

Fig. 3. Duration of dialysis and utility at transplant.

beginning of the simulation for the waiting time. ${ }^{16}$ We then ranked compatible patients based on their expected utility.

In the last step of the simulation, a kidney is assigned to the first patient in the list based on the duration of dialysis or the computed utility. Once the transplant is performed, the patient exits from the pool of transplant candidates. The transplanted patient is then replaced with a "new" patient drawn randomly from the initial patients' database. Each simulation run is a stream of 260 transplants, which equates to a year of transplants, given the average frequency of arrival of kidneys to the transplant centre.

In Fig. 3, we present the utility and duration of dialysis at each transplant along the simulated year. Each point in the graphed lines is obtained as the average over 100 simulation runs. In panel (a), we show that under the benchmark protocol, patients with an extremely long duration of dialysis are, on average, transplanted early in the year. In contrast, under the preference-based protocol, there is no substantial difference across the years; on average, the duration of dialysis at each transplant is 3.38 years. The difference between the two protocols fades away more or less after six months. This is because, in the last step of the simulation round, we kept the waiting list full: once a patient is transplanted, they are replaced by another drawn randomly from the original pool.

As we showed in Fig. 1, duration of dialysis longer than five years is infrequent. Therefore, as the simulation goes on, patients with longer duration of dialysis are replaced by "average" patients and the way compatible patients are ranked becomes less and less relevant. In panel (b), we show that the utility at each transplant is greater under the preference-based protocol than the benchmark protocol. The difference is particularly sizeable for the first 75 transplants, then it fades away and almost disappears after

\footnotetext{
16 For each kidney in the database, we know the exact date it was offered to Padova Transplant Centre. In order to obtain the time elapsed since the beginning of the simulation, we first computed the average span between two subsequent kidney's arrivals, then we multiplied it by the number of kidney draws already performed since the beginning of the simulation.
}

150 transplants. The key finding from Fig. 3 is that there is substantial heterogeneity among patients' preferences and their waiting times. This generates results that are quite different between the two protocols: when patients in the pool have similar durations of dialysis or similar preferences, as it happens towards the end of the simulation year, differences between the two protocols tend to disappear.

These simulation results certainly do not suggest modifying any existing protocol. However, they provide evidence that is worthy of further investigation into the role patients' preferences could have in kidney allocation protocols, and how they could be correctly elicited in a systematic way.

\section{Conclusion}

In this paper, we elicit the preferences from a population of patients waiting for a kidney transplant by using a DCE. We estimate individual willingness to wait (WTW) parameters for changes in the expected graft survival and risk attributes of deceased donors' organs. Experimental design and econometric specification of the model control as much as possible for restrictions imposed by the underlying utility maximisation framework to reduce confounding effects in the estimation of preferences. The baseline results point to heterogeneity in the patients' time and risk preferences. We then devise a model that accounts for systematic differences in preference parameters due to age and duration of dialysis, both observable characteristics of the patients. Both patients' age and their duration of dialysis are significant predictors of WTW for changes in the attributes of kidney transplantation. Younger patients are willing to wait longer than older patients for a better kidney. Patients with longer duration of dialysis are willing to wait longer for a better organ, however, this needs further investigation. The amount of time that a given patient can expect to spend on the waiting list, which depends on blood type and HLA antibodies, is predictable. Therefore, when compared with others, such patients may develop different time preferences because they have been aware since enrolment on 
Table 7

Correlation between coefficients in WTW space.

\begin{tabular}{lllc}
\hline & Waiting time & Graft survival & Infectious risk \\
\hline Waiting time & 1 & -0.8232 & -0.3323 \\
Graft survival & -0.8232 & 1 & 0.6091 \\
Infectious risk & -0.3323 & 0.6091 & 1 \\
Neoplastic risk & -0.5298 & 0.6524 & 0.6524 \\
\hline
\end{tabular}

the waiting list that their chance of finding a compatible organ is lower.

We run a simulation exercise to understand whether the observed preference heterogeneity might have an impact on the kidney allocation mechanism. We show that a preference-based allocation protocol would produce a different allocation of organs and a sizeable increase in the utility of some patients. The implications of our paper for transplant practice are twofold. First, low-quality kidneys should be assigned to older patients as soon as they join the waiting list. Second, pre-emptive transplantation should be expanded as much as possible by offering low-quality organs which would otherwise be discarded, to patients willing to accept them. Pre-emptive kidney transplant performed before the patient begins dialysis, from a deceased donor organ has several clinical advantages. However, this rarely occurs because the current protocol gives a very large weight to waiting time, thus prioritising patients who are waiting longer for a transplant. Hence, it is unlikely that a kidney would be offered to a patient not on dialysis.

This study suggests that a systematic and standardised patients' preference elicitation could be a useful instrument to improve welfare in organ allocation mechanisms. It is worth noting that elements of preference elicitation are already present in many programs. The UK Living Kidney Sharing Scheme is one of the most successful paired kidney exchange programs in the world. ${ }^{17}$ In this scheme, the maximum age of the (living) donor that the patient is willing to accept is a mandated field on the on-line registration form, as is the maximum number of HLA mismatches. Patients can revise this information every three months (i.e., at every matching run). This simple preference elicitation could be easily introduced also for patients registered in the transplant waiting list. Patients should be clearly instructed on how age or other characteristics of the matching, for which they can express their preferences, influence the expected outcome of the transplant. They should also be allowed to revise their preferences at a regular frequency, at least once or twice a year. In this regard, developing personalised kidney transplant decision aid tool can help patients understand their treatment options and outcomes. Awareness of the existence of patients' cognitive biases, like framing effects, and of the relevance of such intangible aspects in medical decision-making (Redelmeier et al., 1993) should not prevent organ procurement organisations

\footnotetext{
17 Kidneys can also be donated by living donors, who are usually relatives of the patients. Donor-recipient pairs who are incompatible by Human Leucocyte Antigen (HLA) type or ABO blood group and unable to donate directly, one to the other, can register in a paired kidney exchange program to achieve compatible transplants with other pairs. For more information on the UK scheme, see https://www.odt.nhs.uk/living-donation/ uk-living-kidney-sharing-scheme/.
}

from designing preference elicitation mechanisms. However, this will require the adoption of standardised and validated protocols.

\section{Authors' contribution}

Mesfin Genie: methodology; investigation; formal analysis; data curation; writing - original draft, review \& editing; Antonio Nicolò: conceptualisation; methodology; investigation; formal analysis; resources; writing - original draft, review \& editing; project administration; funding acquisition; Giacomo Pasini: conceptualisation; methodology; investigation; formal analysis; writing - original draft, review \& editing.

\section{Conflict of interest}

None declared.

\section{Acknowledgements}

The authors wish to thank Noemi Pace and Lorenzo Rocco for helpful comments; Paolo Pellizzari for helping with the simulation; Giacomo Battiston and Veronica Buizza for excellent assistance in the implementation of the experiment. This project has been completed in collaboration with the team of surgeons of the Transplant Unit of the University of Padova Hospital, particularly Paolo Rigotti and Lucrezia Furian, who collaborate with us on the design of the DCE experiment. The authors are grateful to Mandy Ryan and participants of the HESG at the University of Aberdeen, to Nathalie P. Fleury and participants in the EuHEA in Lausanne, to participants in the EEEAESEM 2017 conference in Lisbon, iHEA 2017 conference in Boston, to seminar participants in Venice, Padova and VIVECopenaghen (DK), and to Sebastian Heidenreich and other participants in the health economics research unit (HERU) stated preference seminar at the University of Aberdeen for their comments. We would also like to thank Daniel Rigby (The University of Manchester) and Jürgen Maurer (Université de Lausanne) for their time, interest, and helpful comments. This work was supported by the "Progetto di Ateneo KIDNEY" from the University of Padua.

\section{Appendix A}

\section{A.1 Correlation between parameters' estimates}

Table 7 presents the estimated correlation coefficients among parameters' estimates of the baseline regression in Table 3. 


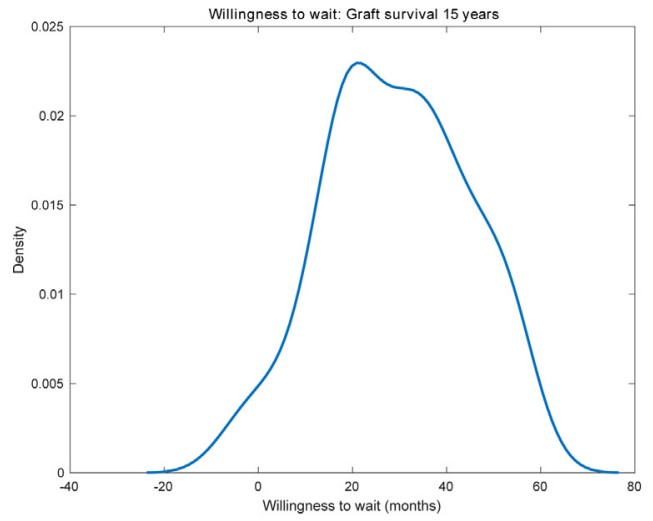

(a) WTW for 15 years of graft survival

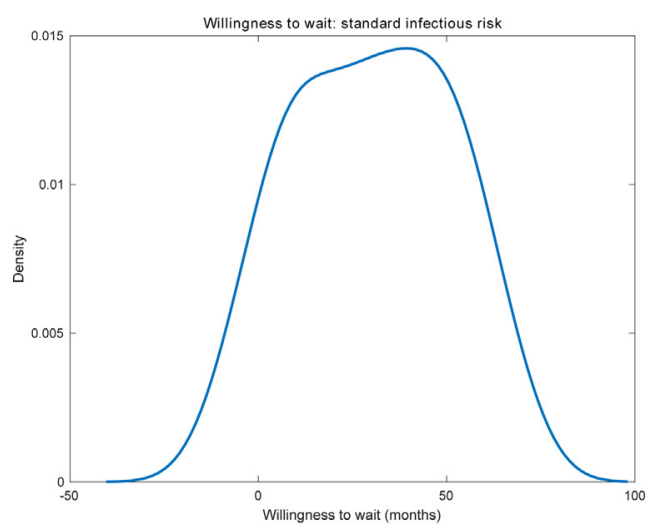

(c) WTW for standard infectious risk

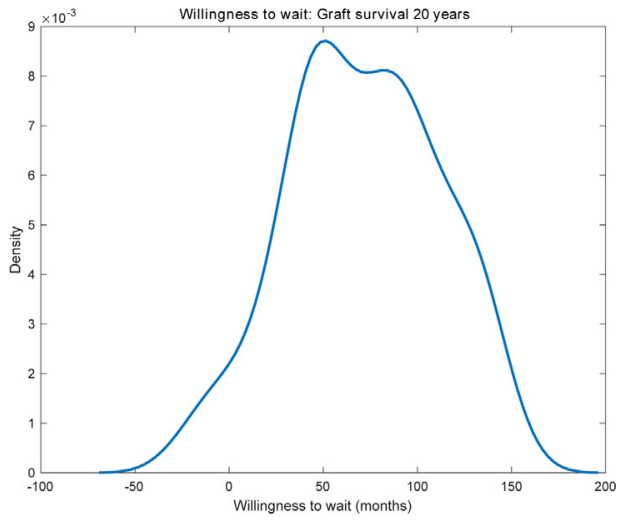

(b) WTW for 20 years of survival

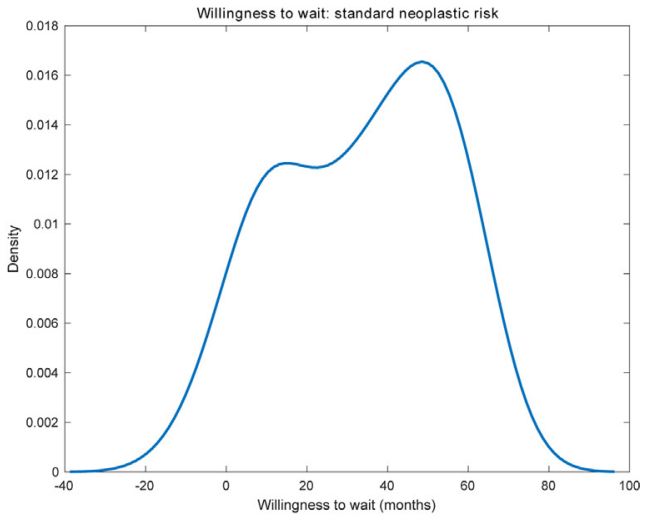

(d) WTW for standard neoplastic risk

Fig. 4. Kernel density plots of the distribution of individual WTW.

\section{A.2 5-Year graft survival differences}

The results are presented in Table 8 and Fig. 4. The variable 'WTW $W_{\text {survival (15 years)' relates to the average WTW for }}$ a kidney that offers 15 years of graft survival rather than 10 years. The benchmark for comparison is an organ offering 10 years of graft survival. On average, patients are willing to wait, ceteris paribus, 29 months longer for a kidney that will offer 15 years of graft survival rather than 10 years.

The WTW for a kidney that will offer 20 years of graft survival is about 71 months longer that for a kidney offering 10 years of graft survival. When the expected graft survival changes from 15 to 20 years, the WTW increases by 42 months. This is consistent with the WTW of 7.52 months for one additional year of graft survival.

In the model recalibrated for a 5-year difference in the expected graft survival (Table 8 ), we find that patients are willing to wait 30 months longer for a kidney of standard infectious risk rather than augmented risk, with all other factors remaining constant. Further, patients are willing to wait 33 months longer for a kidney of standard neoplastic risk rather than augmented neoplastic risk.

The distribution of WTW for 15 years of expected graft survival presented in panel $4 \mathrm{a}$ indicates heterogeneity in
Table 8

Multinomial mixed logit in WTW-space model, empirical distributions first and second moment (normal, waiting time-log-normal).

\begin{tabular}{|c|c|c|}
\hline & $\begin{array}{l}(1) \\
\text { (Mean) }\end{array}$ & $\begin{array}{l}(2) \\
(\mathrm{SD})\end{array}$ \\
\hline Waiting time $(\lambda)$ & $\begin{array}{l}-2.628^{* *} \\
(0.085)\end{array}$ & $\begin{array}{l}2.259 \\
(1.234)\end{array}$ \\
\hline$W_{T W}$ survival $(15$ years $)$ & $\begin{array}{l}29.371^{* *} \\
(2.593)\end{array}$ & $\begin{array}{l}18.337^{* *} \\
(2.718)\end{array}$ \\
\hline$W_{T W}$ survival $(20$ years $)$ & $\begin{array}{l}71.708^{* * * *} \\
(4.625)\end{array}$ & $\begin{array}{l}48.572^{* * * *} \\
(4.519)\end{array}$ \\
\hline$W_{\text {Standard infectious risk }}$ & $\begin{array}{l}30.128^{* * * *} \\
(2.022)\end{array}$ & $\begin{array}{l}31.146^{* * *} \\
(7.713)\end{array}$ \\
\hline$W_{\text {TWtandard neoplastic risk }}$ & $\begin{array}{l}33.479^{* * * *} \\
(2.333)\end{array}$ & $\begin{array}{l}40.471^{* * * *} \\
(16.558)\end{array}$ \\
\hline ASC & $\begin{array}{l}3.191^{* * * *} \\
(0.657)\end{array}$ & - \\
\hline \multicolumn{3}{|l|}{ Model diagnostics } \\
\hline Log-likelihood (LL) at convergence & -2026.513 & \\
\hline McFadden's pseudo- $R^{2}$ & 0.234 & \\
\hline Akaike Information Criterion (AIC)/n & 1.075 & \\
\hline Bayesian Information Criterion (BIC) $/ n$ & 1.118 & \\
\hline$n$ (observations) & 3818 & \\
\hline$r$ (respondents) & 248 & \\
\hline
\end{tabular}

Standard errors in parentheses; ${ }^{*} p<0.1,{ }^{* *} p<0.05,{ }^{* * *} p<0.01$.

Abbreviations: ASC: alternative specific constant, SD: standard deviation. 
Table 9

Results from models in the preference space.

\begin{tabular}{|c|c|c|c|c|c|c|c|}
\hline & \multirow{2}{*}{$\begin{array}{l}\text { Model } 1 \\
\text { Mean }\end{array}$} & \multicolumn{2}{|l|}{ Model 2} & \multicolumn{2}{|l|}{ Model 3} & \multicolumn{2}{|l|}{ Model 4} \\
\hline & & Mean & SD & Mean & SD & Mean & SD \\
\hline Waiting time & $\begin{array}{l}-0.0330^{* * *} \\
(0.0020)\end{array}$ & $\begin{array}{l}-0.0539^{* * * *} \\
(0.0030)\end{array}$ & & $\begin{array}{l}-2.9985^{* * *} \\
(0.1040)\end{array}$ & $\begin{array}{l}1.0645^{* * *} \\
(0.0926)\end{array}$ & $\begin{array}{l}-2.9905^{\text {**** }} \\
(0.1044)\end{array}$ & $\begin{array}{l}2.2114^{*} \\
(1.1404)\end{array}$ \\
\hline Graft survival & $\begin{array}{l}0.1851^{* * * *} \\
(0.0185)\end{array}$ & $\begin{array}{l}0.3042^{* * * *} \\
(0.0318)\end{array}$ & $\begin{array}{l}0.2890^{* * * *} \\
(0.0266)\end{array}$ & $\begin{array}{l}0.3855^{* * * *} \\
(0.0319)\end{array}$ & $\begin{array}{l}0.2435^{* * * *} \\
(0.0329)\end{array}$ & $\begin{array}{l}0.3840^{* * * *} \\
(0.0323)\end{array}$ & $\begin{array}{l}0.1601^{* * * *} \\
(0.0404)\end{array}$ \\
\hline Standard infectious risk & $\begin{array}{l}0.9407^{* * *} \\
(0.0581)\end{array}$ & $\begin{array}{l}1.5446^{* * * *} \\
(0.1270)\end{array}$ & $\begin{array}{l}1.4615^{* * *} \\
(0.1051)\end{array}$ & $\begin{array}{l}1.7532^{* * * *} \\
(0.1207)\end{array}$ & $\begin{array}{l}1.1940^{* * * *} \\
(0.1083)\end{array}$ & $\begin{array}{l}1.7592^{* * *} \\
(0.1317)\end{array}$ & $\begin{array}{l}1.2999 * * * \\
(0.1494)\end{array}$ \\
\hline Standard neoplastic risk & $\begin{array}{l}0.9948^{* * *} \\
(0.0778)\end{array}$ & $\begin{array}{l}1.60966^{* * *} \\
(0.1334)\end{array}$ & $\begin{array}{l}1.2584^{* * *} \\
(0.1223)\end{array}$ & $\begin{array}{l}1.9049^{* * * *} \\
(0.1396)\end{array}$ & $\begin{array}{l}1.1670^{* * *} \\
(0.1341)\end{array}$ & $\begin{array}{l}1.8927^{* * * *} \\
(0.1403)\end{array}$ & $\begin{array}{l}1.1571^{* * * *} \\
(0.1601)\end{array}$ \\
\hline ASC & $\begin{array}{l}0.0920^{* * *} \\
(0.0348)\end{array}$ & $\begin{array}{l}0.1710^{* * * *} \\
(0.0435)\end{array}$ & & $\begin{array}{l}0.1410^{* * * *} \\
(0.0472)\end{array}$ & & $\begin{array}{l}0.1310^{* * * *} \\
(0.0449)\end{array}$ & \\
\hline \multicolumn{8}{|l|}{ Model diagnostics } \\
\hline Log-likelihood (LL) at convergence & -2415.3740 & -2169.0419 & & -2031.2868 & & -1997.7720 & \\
\hline McFadden's pseudo- $R^{2}$ & 0.0868 & 0.1800 & & 0.2320 & & 0.2447 & \\
\hline Akaike Information Criterion (AIC) $/ n$ & 1.2679 & 1.1404 & & 1.0688 & & 1.0565 & \\
\hline Bayesian Information Criterion (BIC)/n & 1.2761 & 1.1535 & & 1.0835 & & 1.0875 & \\
\hline$n$ (observations) & 3818 & 3818 & & 3818 & & 3818 & \\
\hline$r$ (respondents) & 248 & 248 & & 248 & & 248 & \\
\hline
\end{tabular}

Standard errors in parentheses; ${ }^{*} p<0.1,{ }^{* *} p<0.05,{ }^{* * *} p<0.01$.

ASC: alternative specific constant, SD: standard deviation.

Table 10

WTW in the preference space (months).

\begin{tabular}{lllll}
\hline & Model 1 & Model 2 & Model 3 & Model 4 \\
\hline Graft survival & 5.61 & 5.64 & 7.73 & 7.68 \\
Standard infectious risk & 28.51 & 28.66 & 35.13 & 35.18 \\
Standard neoplastic risk & 30.15 & 29.86 & 38.17 & 37.85 \\
\hline
\end{tabular}

WTW: the distribution is concentrated around 20 months. In Fig. 4b, the distributions are more dispersed compared to Fig. 4a, indicating that there is more heterogeneity in the WTW for 20 years of graft survival than for 15 years.

\section{A.3 Sensitivity to modelling choices}

In this section, we run a number of different specifications along the lines of Hole and Kolstad (2012).

\section{A.3.1 Estimates in preference space}

In Table 9 and Table 10, model 1 is a simple multinomial logit model and model 2 is a mixed logit model with independent (zero-correlations) random coefficients for all the attributes except ASC and waiting time. These two models are included as benchmark specifications as they are both common in the DCE literature. Model 3 is equivalent to model 2 except that it allows for preference heterogeneity in terms of waiting time and model 4 also allows for non-zero correlations (correlated random parameters).

\section{A.3.2 Estimates in WTW space}

Model 5 and 6 in Table 11 are similar to models 3 and 4 in the preference space. The coefficients in model 5 are independent (zero-correlation), while the coefficients in model 6 are allowed to be correlated. Model 6 is the baseline specification presented in Table 3. As in the preference space models, the coefficient for waiting time is given a log-normal distribution, and ASC fixed while the rest of the coefficients are normally distributed. In this case, however, the chosen distributions for the attributes graft survival, infectious risk, and neoplastic risk represent the distributions of WTW for these attributes. Both models are estimated using maximum sim-
Table 11

Results from models in the WTW space.

\begin{tabular}{|c|c|c|c|c|}
\hline & \multicolumn{2}{|l|}{ Model 5} & \multicolumn{2}{|l|}{ Model 6} \\
\hline & Mean & SD & Mean & SD \\
\hline Waiting time $(\alpha)$ & $\begin{array}{l}-2.714^{* * *} \\
(0.089)\end{array}$ & $\begin{array}{l}0.932^{* * * *} \\
(0.117)\end{array}$ & $\begin{array}{l}-2.6730^{* * * *} \\
(0.0781)\end{array}$ & $\begin{array}{l}0.8675^{* * * *} \\
(0.1163)\end{array}$ \\
\hline$W_{T W}$ survival & $\begin{array}{l}5.315^{* * *} \\
(0.476)\end{array}$ & $\begin{array}{l}4.694^{* * *} \\
(0.412)\end{array}$ & $\begin{array}{l}7.5155^{* * * *} \\
(0.5086)\end{array}$ & $\begin{array}{l}5.4212^{* * * *} \\
(0.4965)\end{array}$ \\
\hline WTW $W_{\text {standard infectious risk }}$ & $\begin{array}{l}27.968^{* * * *} \\
(1.994)\end{array}$ & $\begin{array}{l}24.619^{* * *} \\
(1.963)\end{array}$ & $\begin{array}{l}31.4621^{* * * *} \\
(2.1725)\end{array}$ & $\begin{array}{l}26.8146^{* * * *} \\
(2.7327)\end{array}$ \\
\hline WTW $W_{\text {standard neoplastic risk }}$ & $\begin{array}{l}27.670^{* * * *} \\
(2.143)\end{array}$ & $\begin{array}{l}21.017^{* * * *} \\
(2.121)\end{array}$ & $\begin{array}{l}34.5935^{* * *} \\
(2.4002)\end{array}$ & $\begin{array}{l}26.5408^{* * * *} \\
(3.1754)\end{array}$ \\
\hline ASC & $\begin{array}{l}3.477^{* * * *} \\
(0.699)\end{array}$ & & $\begin{array}{l}3.0697^{* * * *} \\
(0.6038)\end{array}$ & \\
\hline \multicolumn{5}{|l|}{ Model diagnostics } \\
\hline $\begin{array}{l}\text { Log-likelihood (LL) at } \\
\text { convergence }\end{array}$ & -2134.741 & & -2039.912 & \\
\hline McFadden's pseudo- $R^{2}$ & 0.193 & & 0.2288 & \\
\hline $\begin{array}{l}\text { Akaike Information } \\
\text { Criterion (AIC) } / n\end{array}$ & 1.1224 & & 1.0785 & \\
\hline $\begin{array}{l}\text { Bayesian Information } \\
\text { Criterion }(\mathrm{BIC}) / n\end{array}$ & 1.139 & & 1.109 & \\
\hline$n$ (observations) & 3818 & & 3818 & \\
\hline$r$ (respondents) & 248 & & 248 & \\
\hline
\end{tabular}

Standard errors in parentheses; ${ }^{*} p<0.1,{ }^{* *} p<0.05,{ }^{* * *} p<0.01$.

ASC: Alternative Specific Constant, SD: standard deviation.

ulated likelihood techniques, using 10,000 scrambled Sobol draws.

The coefficients can readily be interpreted as marginal WTW for attribute levels. Our model assumes that all parameters, except for ASC and waiting time, are normally distributed, hence the estimate of mean and standard deviation are provided. Highly statistically significant standard deviations obtained for all the transplant attributes in the mixed logit (MXL) models indicate that the data exhibits considerable heterogeneity of preferences.

It is evident from models 5 and 6 that the means of the WTW estimates are lower than those derived from the corresponding models in the preference space. In Fig. 5, the whole distribution of individual WTW estimates for models 3-6 are shown. It is clear that models estimated in the WTW space (model 5 and 6 ) exhibit distributions with substantially narrower supports. 


\section{A.3.3 Plots-posterior estimates of individual WTW}

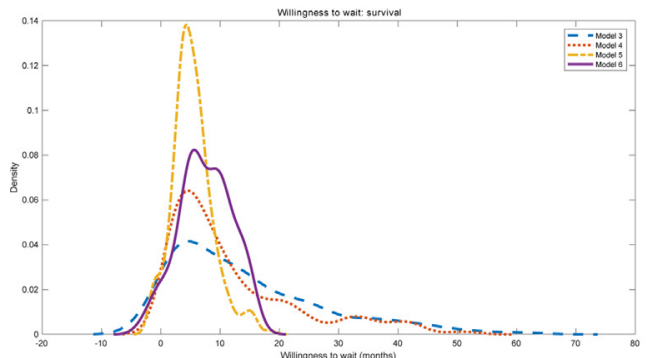

(a) WTW for extra year of survival

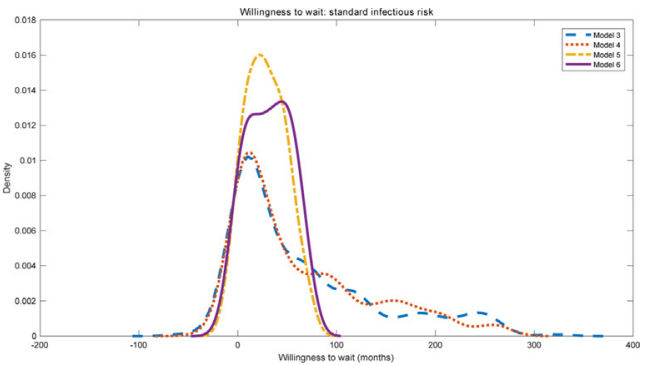

(b) WTW for standard infectious risk

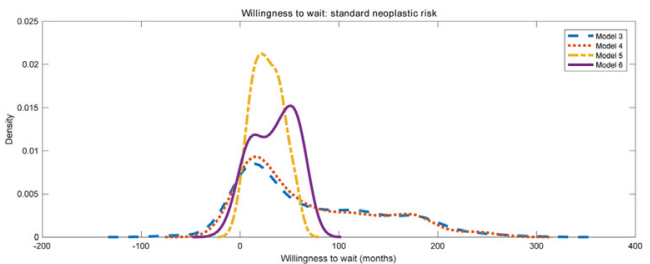

(c) WTW for standard neoplastic risk

Fig. 5. Kernel density plots of posterior estimates of individual WTW.

\section{A.4 Assuming log-normal preference parameters' distribution}

One limitation of assuming normal distribution is that it is unbounded. This implies that nothing prevents obtaining negative WTW on some attributes which one would assume should always be positive, and that a small proportion of patients would have unreasonably high or low WTW for certain attributes.
Table 12 is based on the assumption that the attributes' coefficients are log-normally distributed. Waiting time is entered as negative since the log-normal distribution implies a positive coefficient. We assume that the alternative specific constant (ASC) is fixed. We rerun the models presented in Appendix A.3 assuming lognormality: results in Table 12 refer to models estimated in the preference space, and Table 13 reports models estimated in the WTW space. Model 1 is a simple multi-

Table 12

Results from models in the preference space-all attributes log-normally distributed, ASC fixed.

\begin{tabular}{|c|c|c|c|c|c|c|c|}
\hline & \multirow{2}{*}{$\begin{array}{l}\text { Model } 1 \\
\text { Mean }\end{array}$} & \multicolumn{2}{|l|}{ Model 2} & \multicolumn{2}{|l|}{ Model 3} & \multicolumn{2}{|l|}{ Model 4} \\
\hline & & Mean & SD & Mean & SD & Mean & SD \\
\hline Waiting time & $\begin{array}{l}-0.0330^{* * *} \\
(0.0020)\end{array}$ & $\begin{array}{l}-0.0545^{* *} \\
(0.0025)\end{array}$ & & $\begin{array}{l}-2.9715^{* *} \\
(0.0967)\end{array}$ & $\begin{array}{l}1.0358^{* * *} \\
(0.0970)\end{array}$ & $\begin{array}{l}-2.9262^{* *} \\
(0.1078)\end{array}$ & $\begin{array}{l}1.1004^{* *} \\
(0.1267)\end{array}$ \\
\hline Graft survival & $\begin{array}{l}0.1851^{* * * *} \\
(0.0185)\end{array}$ & $\begin{array}{l}-1.4667^{* * *} \\
(0.1576)\end{array}$ & $\begin{array}{l}0.8798^{* *} \\
(0.0993)\end{array}$ & $\begin{array}{l}-1.1165^{* * *} \\
(0.1109)\end{array}$ & $\begin{array}{l}0.6670^{* * *} \\
(0.0877)\end{array}$ & $\begin{array}{l}-1.0367^{* * *} \\
(0.1087)\end{array}$ & $\begin{array}{l}0.6258^{* *} \\
(0.1215)\end{array}$ \\
\hline Standard infectious risk & $\begin{array}{l}0.9407^{* * * *} \\
(0.0581)\end{array}$ & $\begin{array}{l}0.1051 \\
(0.1244)\end{array}$ & $\begin{array}{l}1.0898^{* * *} \\
(0.1124)\end{array}$ & $\begin{array}{l}0.3685^{* * * *} \\
(0.0826)\end{array}$ & $\begin{array}{l}0.7631^{* *} \\
(0.0793)\end{array}$ & $\begin{array}{l}0.3728^{* * * *} \\
(0.0939)\end{array}$ & $\begin{array}{l}0.9268^{* *} \\
(0.1019)\end{array}$ \\
\hline Standard neoplastic risk & $\begin{array}{l}0.9948^{* * *} \\
(0.0778)\end{array}$ & $\begin{array}{l}0.2465^{* *} \\
(0.1334)\end{array}$ & $\begin{array}{l}0.8869 * * * \\
(0.1144)\end{array}$ & $\begin{array}{l}0.5002^{* * *} \\
(0.0897)\end{array}$ & $\begin{array}{l}0.6450^{* * *} \\
(0.0832)\end{array}$ & $\begin{array}{l}0.5159^{* * *} \\
(0.1002)\end{array}$ & $\begin{array}{l}0.7978 * * * \\
(0.1121)\end{array}$ \\
\hline ASC & $\begin{array}{l}0.0920 \text { *** } \\
(0.0348)\end{array}$ & $\begin{array}{l}0.1696^{* *} \\
(0.0433)\end{array}$ & & $\begin{array}{l}0.1325^{* *} \\
(0.0450)\end{array}$ & & $\begin{array}{l}0.1411^{* * * *} \\
(0.0467)\end{array}$ & \\
\hline \multicolumn{8}{|l|}{ Model diagnostics } \\
\hline Log-likelihood (LL) at convergence & -2415.3740 & -2151.3417 & & -2027.3870 & & -1991.2246 & \\
\hline McFadden's pseudo- $R^{2}$ & 0.0868 & 0.1866 & & 0.2335 & & 0.2472 & \\
\hline Akaike Information Criterion (AIC)/n & 1.2679 & 1.1311 & & 1.0667 & & 1.0530 & \\
\hline Bayesian Information Criterion (BIC) $/ n$ & 1.2761 & 1.1442 & & 1.0815 & & 1.0841 & \\
\hline$n$ (observations) & 3818 & 3818 & & 3818 & & 3818 & \\
\hline$r$ (respondents) & 248 & 248 & & 248 & & 248 & \\
\hline
\end{tabular}

Standard errors in parentheses; ${ }^{*} p<0.1,{ }^{* *} p<0.05,{ }^{* * *} p<0.01$.

ASC: Alternative Specific Constant, SD: standard deviation. 
Table 13

Results from models in the WTW space all attributes log-normally distributed, ASC fixed.

\begin{tabular}{|c|c|c|c|c|}
\hline & \multicolumn{2}{|l|}{ Model 5} & \multicolumn{2}{|l|}{ Model 6} \\
\hline & Mean & SD & Mean & SD \\
\hline $\ln ($ Waiting time $(\alpha))$ & $\begin{array}{l}-2.758^{* * *} \\
(0.1108)\end{array}$ & $\begin{array}{l}1.0887^{* * *} \\
(0.1310)\end{array}$ & $\begin{array}{l}-2.9378^{* * *} \\
(0.1064)\end{array}$ & $\begin{array}{l}1.0534 * * * \\
(0.1252)\end{array}$ \\
\hline $\ln \left(W T W_{\text {survival }}\right)$ & $\begin{array}{l}1.3091^{* * * *} \\
(0.1214)\end{array}$ & $\begin{array}{l}0.9189^{* * *} \\
(0.930)\end{array}$ & $\begin{array}{l}1.8848^{* * *} \\
(0.0990)\end{array}$ & $\begin{array}{l}1.0238^{* * *} \\
(0.0880)\end{array}$ \\
\hline $\ln \left(W T W_{\text {standard infectious risk }}\right)$ & $\begin{array}{l}2.9710^{* * * *} \\
(0.1026)\end{array}$ & $\begin{array}{l}1.1312^{* * *} \\
(0.1072)\end{array}$ & $\begin{array}{l}3.3014 * * * \\
(0.1064)\end{array}$ & $\begin{array}{l}1.3685^{* * *} \\
(0.1231)\end{array}$ \\
\hline $\ln \left(W T W_{\text {standard }}\right.$ neoplastic risk $)$ & $\begin{array}{l}3.0379 * * * \\
(0.1136)\end{array}$ & $\begin{array}{l}1.0259 * * * \\
(0.1206)\end{array}$ & $\begin{array}{l}3.4381^{* * *} \\
(0.1128)\end{array}$ & $\begin{array}{l}1.2989 * * * \\
(0.1397)\end{array}$ \\
\hline ASC & $\begin{array}{l}2.3264^{* * *} \\
(0.5676)\end{array}$ & & $\begin{array}{l}1.1218^{* *} \\
(0.5568)\end{array}$ & \\
\hline \multicolumn{5}{|l|}{ Model diagnostics } \\
\hline Log-likelihood (LL) at convergence & -2095.7754 & & -1993.8122 & \\
\hline McFadden's pseudo- $R^{2}$ & 0.2077 & & 0.2462 & \\
\hline Akaike Information Criterion (AIC)/n & 1.1026 & & 1.0544 & \\
\hline Bayesian Information Criterion (BIC) $/ n$ & 1.1173 & & 1.0855 & \\
\hline$n$ (observations) & 3818 & & 3818 & \\
\hline$r$ (respondents) & 248 & & 248 & \\
\hline
\end{tabular}

Standard errors in parentheses; ${ }^{*} p<0.1,{ }^{* *} p<0.05,{ }^{* * *} p<0.01$.

ASC: alternative specific constant, SD: standard deviation.

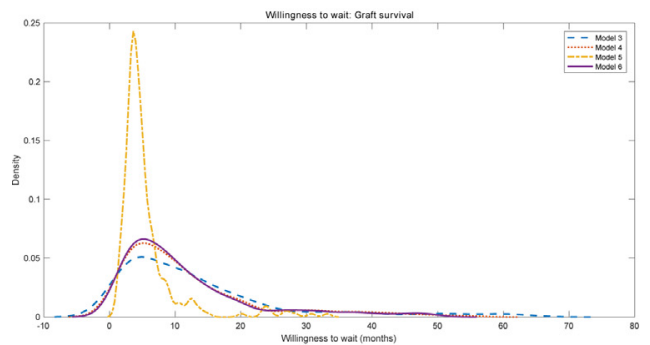

(a) WTW for extra year of survival

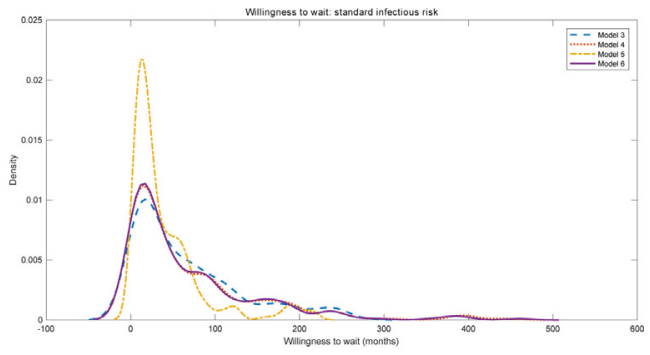

(b) WTW for standard infectious risk

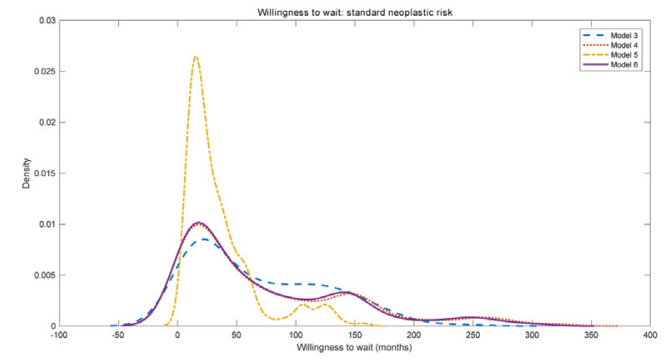

(c) WTW for standard neoplastic risk

Fig. 6. Kernel density plots of posterior estimates of individual WTW, logn.

nomial logit model, and model 2 is a mixed logit model with independent (zero-correlations) random coefficients for all attributes except ASC and waiting time. Model 3 gives estimation results in the preference space with uncorrelated coefficients. Model 4 accounts for full correlation.

The drawback of fitting the preferences to a log-normal distribution is the resulting thicker right-hand tail with unreasonable WTW values (Fig. 6).

\section{Appendix B. WTW and observable characteristics}

In this section, we employ kernel density plots to show the heterogeneity in WTW for changes in the levels of each transplant attribute, and to examine how WTW varies with observable characteristics. We also present the cumulative density functions (CDF) of WTW estimates to describe variations in the WTW in terms of the first-order and second-order stochastic dominance approach. 


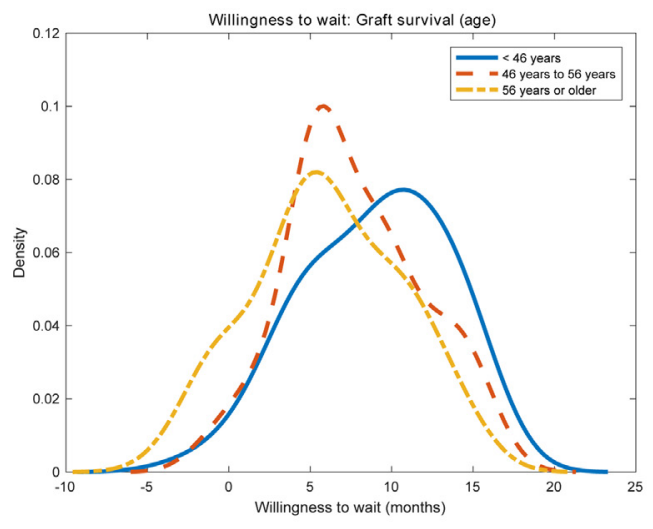

(a) WTW for extra year of survival

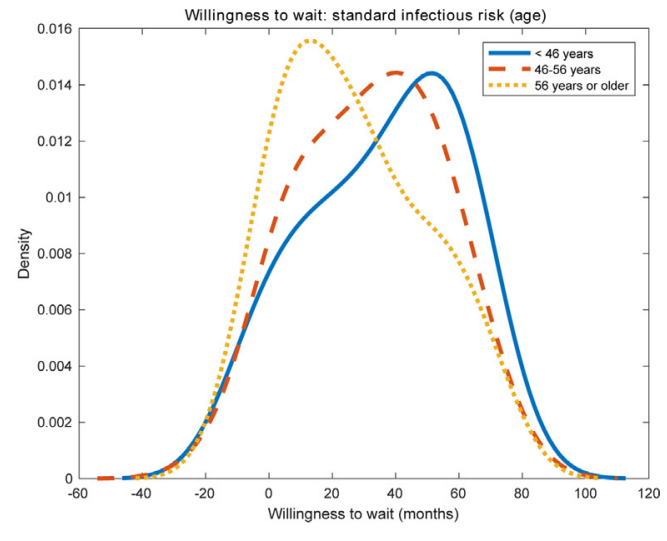

(b) WTW for standard infectious risk

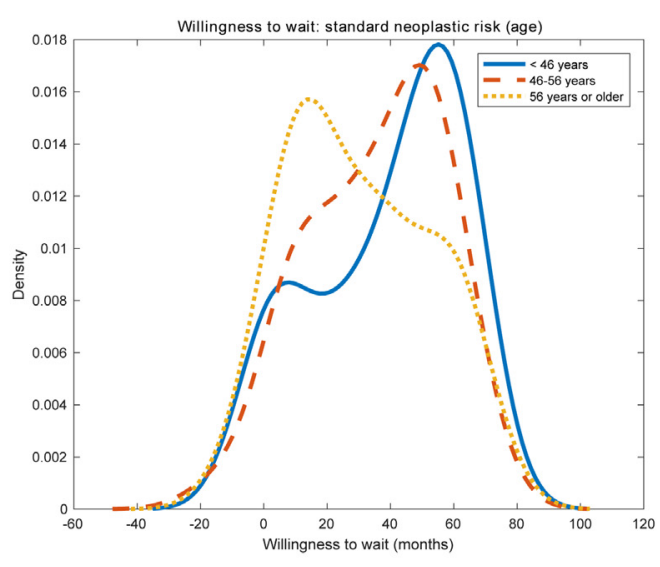

(c) WTW for standard neoplastic risk

Fig. 7. Kernel density plots of the distribution of WTW: effect of age.

\section{B.1 WTW distributions across age groups}

Fig. 7 presents the distributions of the WTW estimates for each of the three attributes across age groups.

The plots are generated using estimates in Table 4 for three age groups: younger than 46,46-56, and 56+ years of age. In Fig. 7a, the distributions of WTW for changes in each attribute across the three age groups differ. For patients aged 56 years and above, the entire distribution of WTW for one extra year of graft survival is shifted to the left. The same applies to the risk attributes: in Fig. $7 \mathrm{~b}$ and $\mathrm{c}$, the entire distributions of WTW for a transplant with standard risk attributes among patients of 56 years and above are shifted to the left.

In Fig. 8, we show the cumulative density functions (CDFs) of the WTW for changes in each of the three attributes. The plots demonstrate that the WTW for each attribute among patients in the first two age groups (i.e., younger than 46 and 46-56 years of age) first-order stochastically dominates the older groups (+56 years). There is evidence that for a given initial level of WTW, the probability that WTW exceeds the initial WTW is higher among the younger patients than the older ones. For exam- ple, given an average WTW for standard infectious risk of 31 months, the probability that WTW exceeds 31 months is higher among the younger patients than the older ones, suggesting that an increase in age is expected to shift the distribution of WTW to the left, thus producing a lower WTW. This implies that keeping a patient on the waiting list as age increases may alter preferences and, hence, the WTW. Accounting for the dynamics in preferences and WTW as age increases, however, would necessitate observing a patient at two points in time.

\section{B.2 WTW distribution and duration of dialysis}

We also present the differences in the shape of the distribution of WTW across three groups of patients according to the duration of dialysis: those who have spent less than 3 years on dialysis, those who have spent 3-10 years on dialysis, and those who have spent over 10 years on dialysis. The data reveals that $58.87 \%$ (146 patients) had spent less than 3 years, $33.47 \%$ (83 patients) had spent 3-10 years, and the remaining $7.66 \%$ (19 patients) had spent above 10 


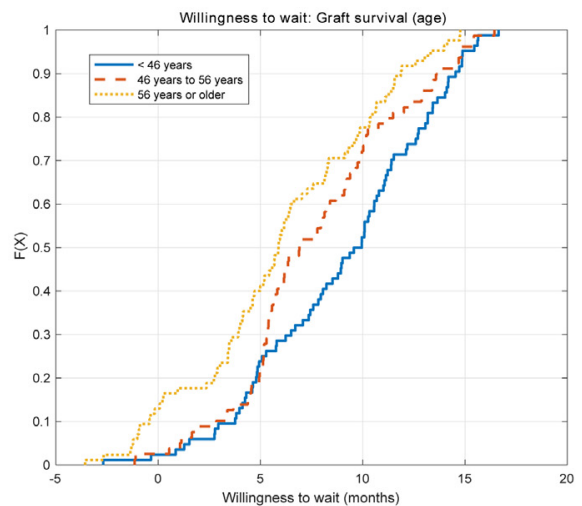

(a) WTW (months) for extra year of survival

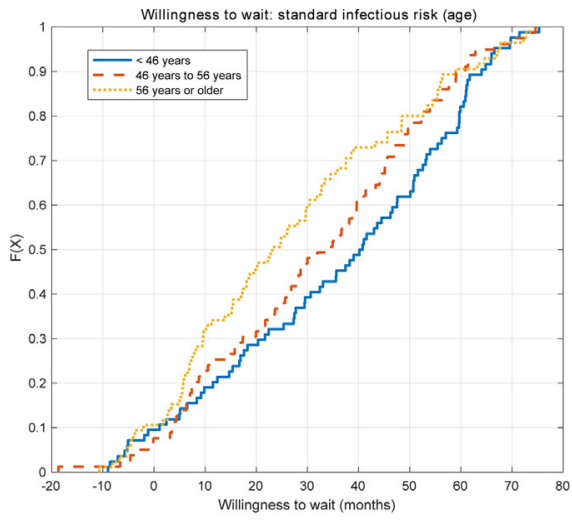

(b) WTW (months) for standard infectious risk

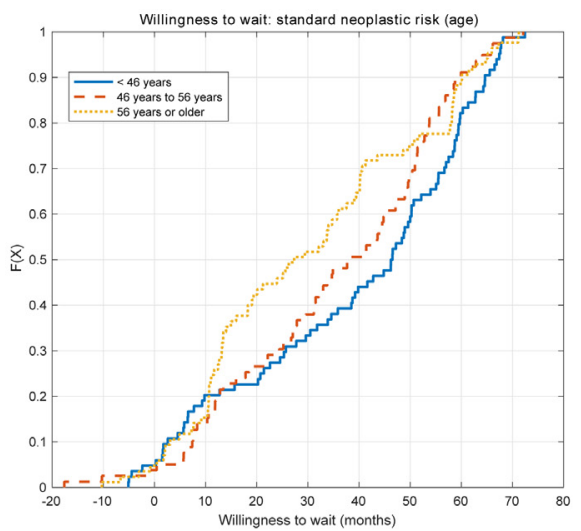

(c) WTW (months) for standard neoplastic risk

Fig. 8. Visual representations of the CDF of WTW values: effect of age.

years on dialysis. ${ }^{18}$ The shapes of the distributions of the WTW are different across patients with a different duration of dialysis (Fig. 9). The distributions of WTW for changes in each of the attributes are shifted to the left among patients with a duration of dialysis of over ten years. For patients with over 10 years of dialysis, there is a lower frequency at the mean but a wider distribution elsewhere, implying more heterogeneity in the WTW values. While the dispersions are roughly the same for standard infectious risk and standard neoplastic risk, the distribution of WTW for a kidney that will offer an extra year of graft survival is more concentrated. For patients with less than three years on dialysis, however, the distributions are shifted to the left for all the attributes, suggesting the presence of impatience (time-discounting) predominantly in the early stages of dialysis.

In Fig. 10, we show the CDF of WTW for changes in each of the three attributes. The CDF of WTW for changes in each attribute among patients with duration of dialysis of over

\footnotetext{
18 We repeated the analysis dividing the population in tertiles of the distribution of time in dialysis, and result are consistent with what we present here.
}

three years first-order stochastically dominates patients with less than three years. At any initial level of WTW, the probability that WTW exceeds the initial level of WTW is higher among patients with over three years of dialysis. For example, panel 10a of Fig. 10 suggests that given the WTW of 5 months for a kidney that will offer an additional year of functioning, the probability that the WTW exceeds 5 months is higher among patients with duration of dialysis of 3-10 years and over ten years compared to patients with less than three years on dialysis.

\section{Appendix C}

In what follows, the English translation of instructions and the questionnaire are presented.

\section{C.1 Kidney transplant survey (original in Italian)}

I am part of a group of researchers from the University of Padua and the Ca' Foscari University of Venice carrying out a study that aims to assess whether it is possible to increase the well-being of patients who need a kidney transplant, naturally maintaining or by improving the clinical results of transplants. This research project, considered of strategic 


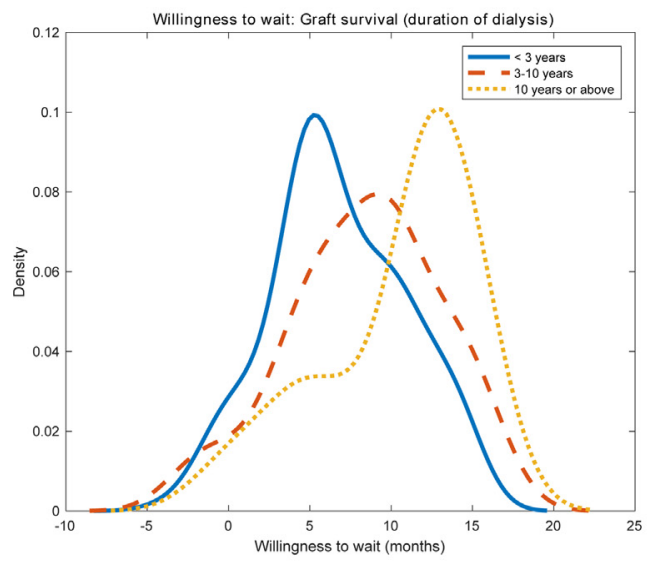

(a) WTW for extra year of survival

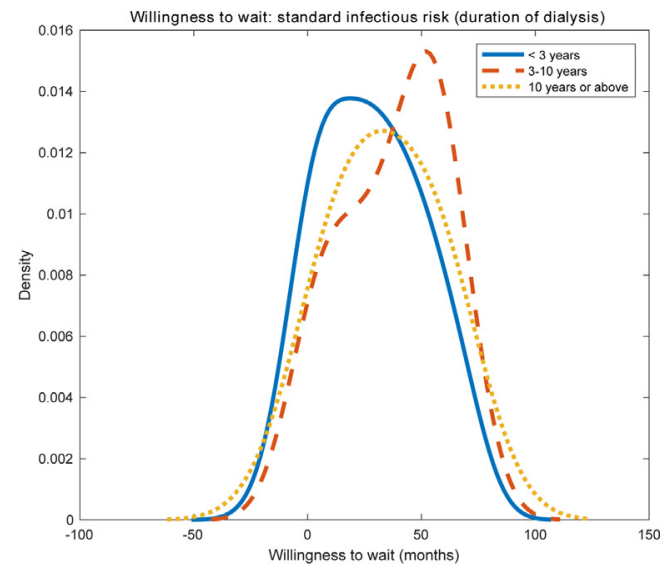

(b) WTW for standard infectious risk

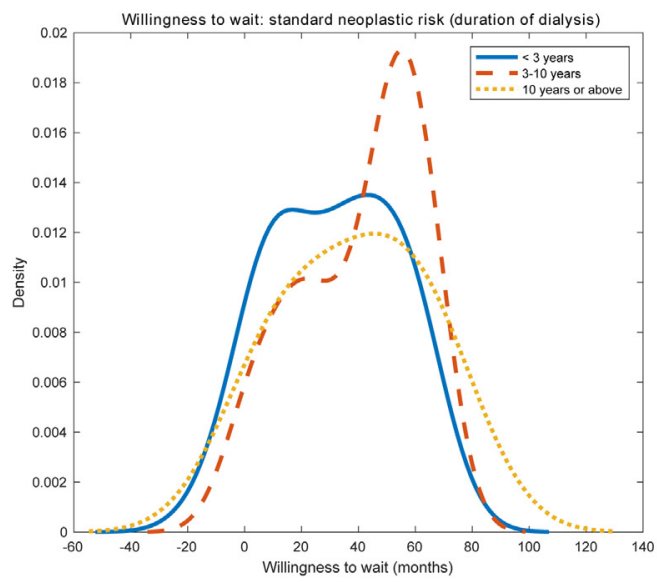

(c) WTW for standard neoplastic risk

Fig. 9. Kernel density plots of the distribution of WTW: effect of dialysis duration.

importance by the University of Padua, provides a survey on the characteristics and preferences of patients awaiting kidney transplantation. Your participation in this investigation is vital for scientific research. We will ask you about the preferences for alternative pairs of medical treatments, some demographic information, and your general state of health.

The results of this study will be published in specialised scientific journals and presented in scientific conferences. The information collected in this questionnaire will be linked to the information already held by the Regional Transplant Centre, but no publication or presentation will ever contain your name or any information that could identify you. All data collected will be archived and analysed in a strictly anonymous manner, pursuant to art. 7 and of the art. 13 of the Legislative Decree n. 196/03 in force since 1 January 2004 on the protection of individuals concerning the processing of personal data. Furthermore, the use of your data for commercial purposes is strictly prohibited. If you do not have any further questions or requests for clarification, we can start the interview.

\section{C.2 Patients' preferences for the different transplant options}

Instructions: In this section sixteen alternative treatment pairs will be presented. You will be asked to express your preference between treatment $A$ and treatment $B$ by placing an $\mathrm{X}$ in the box below them. We remind you again that the answers will have no influence on how the future kidney transplant will be conducted. A transplant (treatment) is characterised by the following factors:

- Waiting time is the time one will have to wait in order to obtain the proposed transplant. The waiting time depends on the characteristics of the recipient and the frequency with which donors of a particular type are available. 


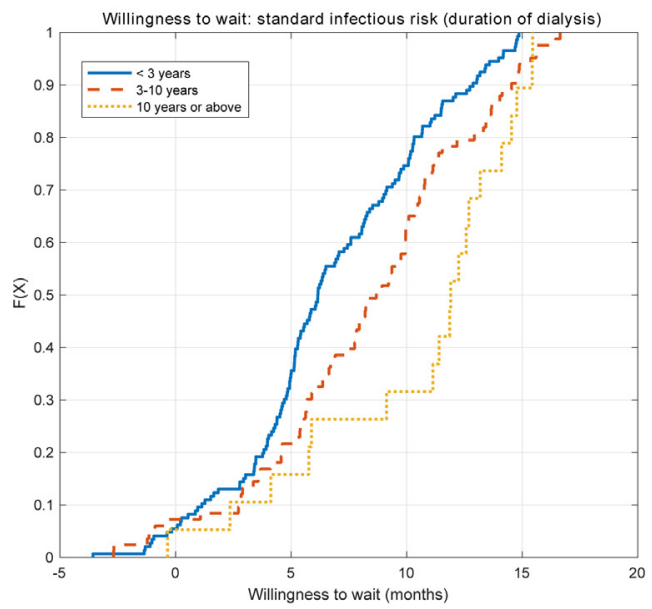

(a) WTW for extra year of survival

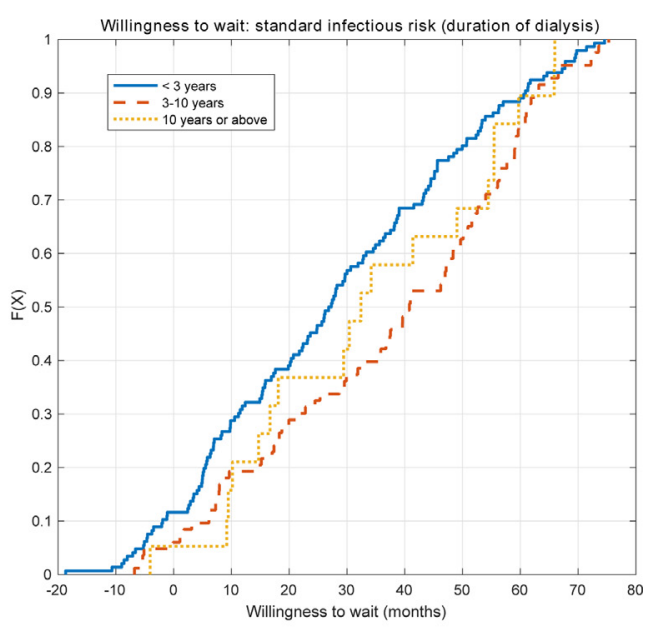

(b) WTW for standard infectious risk

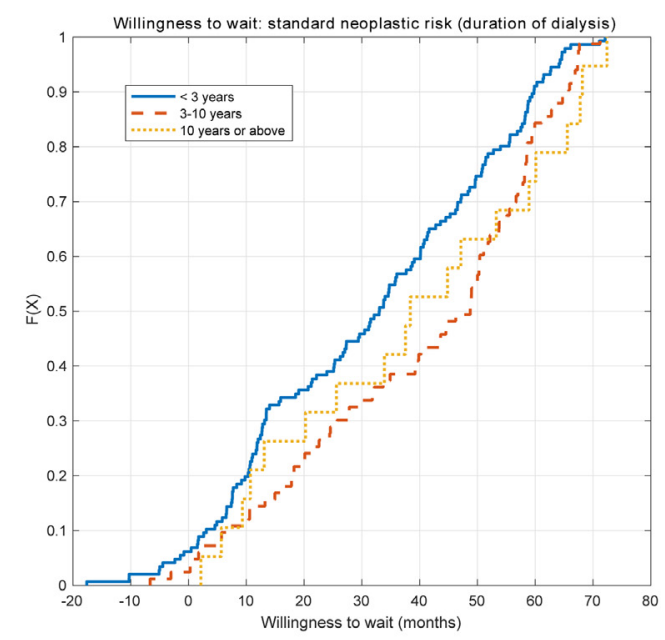

(c) WTW for standard neoplastic risk

Fig. 10. Visual representations of the CDF of WTW values: effect of dialysis duration.

- Graft survival is determined by the characteristics of the transplanted graft, the characteristics of the recipient, and the compatibility between donor and recipient.

- Infectious risk (standard or augmented) is the risk of contracting an infectious disease through the graft. If it is standard, the organ has undergone all the possible checks, even if complete safety cannot be guaranteed. If it is augmented, some of the controls have not been performed, or the donor had some risky behaviours in the days before his or her death, but an infection may still not result from clinical diagnostics (even if it is possible).

- Neoplastic risk (standard or augmented) is the risk of contracting a tumour through the transplanted organ. If it is standard, the donor was not affected by a tumour, almost surely, even if a minimum level of risk does exist (for example, if the donor was not aware of the problem and it did not emerge from checks). It is augmented if the donor had some kinds of neoplastic disease. Still, it is not high in terms of probability, because the due checks have been performed.

Below are proposed 16 pairs of treatments (transplants) described by different attributes. Please, indicate the preferred one for each pair, by crossing $(\mathrm{X})$ in the square below it.

1. Which of the two treatments would you prefer? Put an $\mathrm{X}$ below the chosen treatment

\begin{tabular}{lll} 
& Treatment A & Treatment B \\
\hline Waiting Time & 6 Months & 6 Months \\
Expected Graft Survival & 20 Years & 15 Years \\
Infectious Risk & Standard & Standard \\
Neoplastic Risk & Augmented & Standard \\
Your Choice? & $\square$ & $\square$
\end{tabular}

2. Which of the two treatments would you prefer? Put an $\mathrm{X}$ below the chosen treatment 


\begin{tabular}{lll} 
& Treatment A & Treatment B \\
\hline Waiting Time & 12 Months & 36 Months \\
Expected Graft Survival & 15 Years & 20 Years \\
Infectious Risk & Standard & Augmented \\
Neoplastic Risk & Standard & Augmented \\
Your Choice? & $\square$ & $\square$
\end{tabular}

3. Which of the two treatments would you prefer? Put an $\mathrm{X}$ below the chosen treatment

\begin{tabular}{lll} 
& Treatment A & Treatment B \\
\hline Waiting Time & 60 Months & 6 Months \\
Expected Graft Survival & 20 Years & 15 Years \\
Infectious Risk & Standard & Augmented \\
Neoplastic Risk & Augmented & Standard \\
Your Choice? & $\square$ & $\square$
\end{tabular}

4. Which of the two treatments would you prefer? Put an $\mathrm{X}$ below the chosen treatment

\begin{tabular}{lll} 
& Treatment A & Treatment B \\
\hline Waiting Time & 6 Months & 12 Months \\
Expected Graft Survival & 10 Years & 10 Years \\
Infectious Risk & Augmented & Standard \\
Neoplastic Risk & Augmented & Augmented \\
Your Choice? & $\square$ & $\square$
\end{tabular}

5. Which of the two treatments would you prefer? Put an $\mathrm{X}$ below the chosen treatment

\begin{tabular}{lll} 
& Treatment A & Treatment B \\
\hline Waiting Time & 36 Months & 60 Months \\
Expected Graft Survival & 10 Years & 10 Years \\
Infectious Risk & Augmented & Standard \\
Neoplastic Risk & Standard & Standard \\
Your Choice? & $\square$ & $\square$
\end{tabular}

6. Which of the two treatments would you prefer? Put an $\mathrm{X}$ below the chosen treatment

\begin{tabular}{lll} 
X below the chosen treatment & Treatment A & Treatment B \\
\hline Waiting Time & 60 Months & 36 Months \\
Expected Graft Survival & 15 Years & 10 Years \\
Infectious Risk & Augmented & Augmented \\
Neoplastic Risk & Augmented & Standard \\
Your Choice? & $\square$ & $\square$
\end{tabular}

7. Which of the two treatments would you prefer? Put an $\mathrm{X}$ below the chosen treatment

\begin{tabular}{lll} 
& Treatment A & Treatment B \\
\hline Waiting Time & 60 Months & 60 Months \\
Expected Graft Survival & 20 Years & 20 Years \\
Infectious Risk & Augmented & Standard \\
Neoplastic Risk & Standard & Augmented \\
Your Choice? & $\square$ & $\square$
\end{tabular}

8. Which of the two treatments would you prefer? Put an $\mathrm{X}$ below the chosen treatment

\begin{tabular}{lll} 
& Treatment A & Treatment B \\
\hline Waiting Time & 36 Months & 6 Months \\
Expected Graft Survival & 15 Years & 10 Years \\
Infectious Risk & Standard & Augmented \\
Neoplastic Risk & Augmented & Augmented \\
Your Choice? & $\square$ & $\square$
\end{tabular}

9. Which of the two treatments would you prefer? Put an $\mathrm{X}$ below the chosen treatment

\begin{tabular}{lll} 
& Treatment A & Treatment B \\
\hline Waiting Time & 6 Months & 12 Months \\
Expected Graft Survival & 15 Years & 20 Years \\
Infectious Risk & Standard & Augmented \\
Neoplastic Risk & Standard & Standard \\
Your Choice? & $\square$ & $\square$
\end{tabular}

10. Which of the two treatments would you prefer? Put an $\mathrm{X}$ below the chosen treatment

\begin{tabular}{lll} 
& Treatment A & Treatment B \\
\hline Waiting Time & 12 Months & 60 Months \\
Expected Graft Survival & 10 Years & 15 Years \\
Infectious Risk & Standard & Augmented \\
Neoplastic Risk & Augmented & Augmented \\
Your Choice? & $\square$ & $\square$
\end{tabular}

11. Which of the two treatments would you prefer? Put an $\mathrm{X}$ below the chosen treatment

\begin{tabular}{lll} 
& Treatment A & Treatment B \\
\hline Waiting Time & 12 Months & 36 Months \\
Expected Graft Survival & 20 Years & 20 Years \\
Infectious Risk & Augmented & Standard \\
Neoplastic Risk & Standard & Standard \\
Your Choice? & $\square$ & $\square$
\end{tabular}

12. Which of the two treatments would you prefer? Put an $\mathrm{X}$ below the chosen treatment

\begin{tabular}{lll} 
& Treatment A & Treatment B \\
\hline Waiting Time & 6 Months & 12 Months \\
Expected Graft Survival & 15 Years & 15 Years \\
Infectious Risk & Augmented & Standard \\
Neoplastic Risk & Standard & Standard \\
Your Choice? & $\square$ & $\square$
\end{tabular}

13. Which of the two treatments would you prefer? Put an $\mathrm{X}$ below the chosen treatment

\begin{tabular}{lll} 
& Treatment A & Treatment B \\
\hline Waiting Time & 60 Months & 12 Months \\
Expected Graft Survival & 10 Years & 15 Years \\
Infectious Risk & Standard & Augmented \\
Neoplastic Risk & Standard & Augmented \\
Your Choice? & $\square$ & $\square$
\end{tabular}

14. Which of the two treatments would you prefer? Put an $\mathrm{X}$ below the chosen treatment

\begin{tabular}{lll} 
& Treatment A & Treatment B \\
\hline Waiting Time & 36 Months & 60 Months \\
Expected Graft Survival & 20 Years & 20 Years \\
Infectious Risk & Augmented & Augmented \\
Neoplastic Risk & Augmented & Standard \\
Your Choice? & $\square$ & $\square$
\end{tabular}

15. Which of the two treatments would you prefer? Put an $X$ below the chosen treatment

\begin{tabular}{lll} 
& Treatment A & Treatment B \\
\hline Waiting Time & 36 Months & 6 Months \\
Expected Graft Survival & 20 Years & 20 Years \\
Infectious Risk & Standard & Standard \\
Neoplastic Risk & Standard & Augmented \\
Your Choice? & $\square$ & $\square$
\end{tabular}

16. Which of the two treatments would you prefer? Put an $\mathrm{X}$ below the chosen treatment

\begin{tabular}{lll} 
& Treatment A & Treatment B \\
\hline Waiting Time & 12 Months & 36 Months \\
Expected Graft Survival & 15 Years & 15 Years \\
Infectious Risk & Augmented & Standard \\
Neoplastic Risk & Augmented & Augmented \\
Your Choice? & $\square$ & $\square$
\end{tabular}

We thank you for your precious time and collaboration. Next are a few questions about the logical abilities of patients about different combinations of choices. 


\section{C.3 SHARE Numeracy Questions}

Now I would like to ask you some questions that are needed to evaluate how people use numbers in everyday life.

1. The probability of contracting an illness is 10 percent, how many people out of one thousand would be expected to get the disease?

2. In a sale, a shop is selling all items at half price. Before the sale the sofa costs 300 Euros. How much will it cost in the sale?

3. A second hand car dealer is selling a car for 6,000 Euro. This is two-thirds of what it costs new. How much did the car cost new?

\section{Personal information:}

1. Education

$\square$ Elementary $\square$ Lower middle $\square$ Higher middle $\square$ Degree

2. Family composition (not just the people living with you)

$\square$ Mother $\square$ Father $\square$ Brother/sisters $\square$ Male-No. $-\square$

Female-No. $-\square$ Wife $\square$ Husband $\square$ Cohabiting $\square$ Children

$\square$ Male-No. $-\square$ Female-No.- $\square$ Other

3. What is your current profession?

$\square$ Manager $\square$ Self-employed $\square$ Employee $\square$ Housewife $\square$

Retired $\square$ Student $\square$ Other-

4. Do you currently have a disability persion?

$\square$ Yes $\square$ No

\section{Medical information:}

1. First year diagnosis/age of onset of the pathology-

2. Dialysis start date: month/year-

3. Dialysis type

$\square$ Haemodialysis $\square$ Peritoneal dialysis

4. Presence of diabetes mellitus

$\square$ yes $\square$ no

5. Date listed for renal transplantation: $-/-/-$

\section{Dialysis:}

In your opinion, how true or false are the following statements?

\begin{tabular}{llllll} 
& $\begin{array}{l}\text { Absolutely } \\
\text { True }\end{array}$ & $\begin{array}{c}\text { True I don't } \\
\text { know }\end{array}$ & $\begin{array}{c}\text { False } \\
\text { Fabsolutely }\end{array}$ \\
\hline 1 Dialysis affects my & 1 & 2 & 3 & 4 & 5 \\
$\begin{array}{l}\text { life too much } \\
2\end{array}$ & 1 & 2 & 3 & 4 & 5 \\
$\begin{array}{l}\text { Dialysis makes me } \\
\text { lose too much time }\end{array}$ & 1 & 2 & 3 & 4 & 5 \\
$3 \begin{array}{l}\text { I find it frustrating } \\
\text { to live with dialysis }\end{array}$ & 1 & 2 & 3 & 4 & 5 \\
$4 \begin{array}{l}\text { I feel dialysis a } \\
\text { burden to my }\end{array}$ & 1 & & & &
\end{tabular}

\section{General health status:}

$\square$ Excellent $\square$ Very good $\square$ Good $\square$ Passable $\square$ Poor

\section{References}

Agarwal, N., Ashlagi, I., Rees, M.A., Somaini, P.J., Waldinger, D.C., 2019. An Empirical Framework for Sequential Assignment: The Allocation of Deceased Donor Kidneys. Technical Report. National Bureau of Economic Research.

Aizaki, H., Nishimura, K., 2008. Design and analysis of choice experiments using R: a brief introduction. Agric. Inform. Res. 17 (2), 86-94.

Berry, S., Levinsohn, J., Pakes, A., 1995. Automobile prices in market equilibrium. Econometrica: J. Econ. Soc., 841-890.

Bhat, C.R., 2000. Incorporating observed and unobserved heterogeneity in urban work travel mode choice modeling. Transp. Sci. 34 (2), 228-238.
Bishai, D.M., 2004. Does time preference change with age? J. Popul. Econ. 17 (4), 583-602.

Brown, P., Panattoni, L., Cameron, L., Knox, S., Ashton, T., Tenbensel, T., Windsor, J., 2015. Hospital sector choice and support for public hospital care in New Zealand: results from a labeled discrete choice survey. J. Health Econ. 43, 118-127.

Clark, M.D., Szczepura, A., Gumber, A., Howard, K., Moro, D., Morton, R.L., 2018. Measuring trade-offs in nephrology: a systematic review of discrete choice experiments and conjoint analysis studies. Nephrol. Dial. Transpl. 33 (2), 348-355.

Coast, J., Horrocks, S., 2007. Developing attributes and levels for discrete choice experiments using qualitative methods. J. Health Serv. Res. Policy 12 (1), 25-30.

Czajkowski, M., Budziński, W., 2019. Simulation error in maximum likelihood estimation of discrete choice models. J. Choice Modell. 31 , 73-85.

de Bekker-Grob, E.W., Ryan, M., Gerard, K., 2012. Discrete choice experiments in health economics: a review of the literature. Health Econ. 21 (2), 145-172

Determann, D., Lambooij, M.S., Steyerberg, E.W., de Bekker-Grob, E.W., De Wit, G.A., 2017. Impact of survey administration mode on the results of a health-related discrete choice experiment: online and paper comparison. Value Health 20 (7), 953-960.

Eggers, P., 1992. Comparison of treatment costs between dialysis and transplantation. Seminars in Nephrology, vol. 12. Elsevier, pp. 284-289.

Eggers, P.W., Kucken, L.E., 1994. Cost issues in transplantation. Surg. Clin. N. Am. 74 (5), 1259-1267.

Fischer, B., Telser, H., Zweifel, P., 2018. End-of-life healthcare expenditure: testing economic explanations using a discrete choice experiment. J. Health Econ. 60, 30-38.

Gandolfini, I., Buzio, C., Zanelli, P., Palmisano, A., Cremaschi, E., Vaglio, A., Piotti, G., Melfa, L., La Manna, G., Feliciangeli, G., et al., 2014. The kidney donor profile index (kdpi) of marginal donors allocated by standardized pretransplant donor biopsy assessment: distribution and association with graft outcomes. Am. J. Transpl. 14 (11), 2515-2525.

Greene, W.H., Hensher, D.A., Rose, J., 2006. Accounting for heterogeneity in the variance of unobserved effects in mixed logit models. Transp. Res. Part B: Methodol. 40 (1), 75-92.

Hagemi, A., Plumpton, C., Hughes, D.A., 2017. Renal transplant patients' preference for the supply and delivery of immunosuppressants in wales: a discrete choice experiment. BMC Nephrol. 18 (1), 305.

Hart, A., Smith, J., Skeans, M., Gustafson, S., Wilk, A., Robinson, A., Wainright, J., Haynes, C., Snyder, J., Kasiske, B., Israni, A., 2018. Optn/srtr 2016 annual data report: kidney. Am. J. Transpl. 18 (S1), 18-113.

Hasund, K.P., Kataria, M., Lagerkvist, C.J., 2011. Valuing public goods of the agricultural landscape: a choice experiment using reference points to capture observable heterogeneity. J. Environ. Plann. Manag. $54(1), 31-53$.

Held, J., Philip, F., McCormick, A., Ojo, J., Roberts, P., 2016. A cost-benefit analysis of government compensation of kidney donors. Am. J. Transpl. 16 (3), 877-885.

Hensher, D.A., 2006. How do respondents process stated choice experiments? Attribute consideration under varying information load. J. Appl. Econometr. 21 (6), 861-878.

Hensher, D.A., Greene, W.H., 2011. Valuation of travel time savings in WTP and preference space in the presence of taste and scale heterogeneity. J. Transp. Econ. Policy (JTEP) 45 (3), 505-525.

Hole, A.R., 2008. Modelling heterogeneity in patients preferences for the attributes of a general practitioner appointment. J. Health Econ. 27 (4), 1078-1094.

Hole, A.R., Kolstad, J.R., 2012. Mixed logit estimation of willingness to pay distributions: a comparison of models in preference and WTP space using data from a health-related choice experiment. Empir. Econ. 42 (2), 445-469.

Jay, C.L., Washburn, K., Dean, P.G., Helmick, R.A., Pugh, J.A., Stegall, M.D. 2017. Survival benefit in older patients associated with earlier transplant with high kdpi kidneys. Transplantation 101 (4), 867.

Johnson, F.R., Lancsar, E., Marshall, D., Kilambi, V., Mühlbacher, A., Regier, D.A., Bresnahan, B.W., Kanninen, B., Bridges, J.F., 2013. Constructing experimental designs for discrete-choice experiments: report of the ispor conjoint analysis experimental design good research practices task force. Value Health 16 (1), 3-13.

Kamran, S., Conti, F., Pomey, M.-P., Baron, G., Calmus, Y., Vidal-Trecan, G., 2017. Patients' preferences in transplantation from marginal donors: results of a discrete choice experiment. Transpl. Int. 30 (6), 589-602. 
Lancaster, K.J., 1966. A new approach to consumer theory. J. Polit. Econ., $132-157$.

Lancsar, E., Fiebig, D.G., Hole, A.R., 2017. Discrete choice experiments: a guide to model specification, estimation and software. Pharmacoeconomics 35 (7), 697-716.

Lancsar, E., Wildman, J., Donaldson, C., Ryan, M., Baker, R., 2011. Deriving distributional weights for qalys through discrete choice experiments. J. Health Econ. 30 (2), 466-478.

Marshall, D., Deal, K., Conner-Spady, B., Bohm, E., Hawker, G., Loucks, L., MacDonald, K., Noseworthy, T., 2018. How do patients trade-off surgeon choice and waiting times for total joint replacement: a discrete choice experiment. Osteoarthr. Cartil. 26 (4), 522-530.

McFadden, D., 1974. Conditional logit analysis of qualitative choice behaviour. In: Zerembka, P. (Ed.), Frontiers in Econometrics. Academic Press, New York, pp. 105-142.

McFadden, D., Train, K., 2000. Mixed MNL models for discrete response. J. Appl. Econ., 447-470.

Meenakshi, J., Banerji, A., Manyong, V., Tomlins, K., Mittal, N., Hamukwala, P., 2012. Using a discrete choice experiment to elicit the demand for a nutritious food: willingness-to-pay for orange maize in rural Zambia. J. Health Econ. 31 (1), 62-71.

Merion, R.M., Ashby, V.B., Wolfe, R.A., Distant, D.A., Hulbert-Shearon, T.E., Metzger, R.A., Ojo, A.O., Port, F.K., 2005. Deceased-donor characteristics and the survival benefit of kidney transplantation. JAMA 294 (21), 2726-2733.

Metzger, R.A., Delmonico, F.L., Feng, S., Port, F.K., Wynn, J.J., Merion, R.M., 2003. Expanded criteria donors for kidney transplantation. Am. J. Transpl. 3 (s4), 114-125.

Milte, R., Ratcliffe, J., Chen, G., Crotty, M., 2018. What characteristics of nursing homes are most valued by consumers? A discrete choice experiment with residents and family members. Value Health 21 (7), 843-849.

Morin, R.-A., Suarez, A.F., 1983. Risk aversion revisited. J. Finance 38 (4), $1201-1216$

Nieboer, A.P., Koolman, X., Stolk, E.A., 2010. Preferences for long-term care services: willingness to pay estimates derived from a discrete choice experiment. Soc. Sci. Med. 70 (9), 1317-1325.

Ojo, A.O., Hanson, J.A., Meier-Kriesche, H.-U., Okechukwu, C.N., Wolfe, R.A., Leichtman, A.B., Agodoa, L.Y., Kaplan, B., Port, F.K., 2001. Survival in recipients of marginal cadaveric donor kidneys compared with other recipients and wait-listed transplant candidates. J. Am. Soc. Nephrol. 12 (3), 589-597.

Organ Procurement and Transplantation Network, 2019. A Guide to Calculating and Interpreting the Kidney Donor Profile Index (KDPI)

Patterson, J.A., Holdford, D.A., Harpe, S.E., 2019. Patient preferences for objective quality metrics during community pharmacy selection: a discrete choice experiment. Res. Soc. Administr. Pharmacy 15 (6), 641-649.
Rao, P.S., Schaubel, D.E., Guidinger, M.K., Andreoni, K.A., Wolfe, R.A., Merion, R.M., Port, F.K., Sung, R.S., 2009. A comprehensive risk quantification score for deceased donor kidneys: the kidney donor risk index. Transplantation 88 (2), 231-236.

Redelmeier, D.A., Rozin, P., Kahneman, D., 1993. Understanding patients' decisions: cognitive and emotional perspectives. JAMA 270 (1), $72-76$.

Reese, P.P., Tehrani, T., Lim, M.A., Asch, D.A., Blumberg, E.A., Simon, M.K. Bloom, R.D., Halpern, S.D., 2010. Determinants of the decision to accept a kidney from a donor at increased risk for blood-borne viral infection. Clin. J. Am. Soc. Nephrol., 917-923.

Rigby, D., Alcon, F., Burton, M., 2010. Supply uncertainty and the economic value of irrigation water. Eur. Rev. Agric. Econ. 37 (1), 97-117.

Roth, A.E., Sönmez, T., Ünver, M.U., 2004. Kidney exchange. Quart. J. Econ. 119 (2), 457-488.

Rousseau, S., Rousseau, R., 2012. Interactions between journal attributes and authors' willingness to wait for editorial decisions. J. Am. Soc. Inform. Sci. Technol. 63 (6), 1213-1225.

Ryan, M., Gerard, K., 2003. Using discrete choice experiments to value health care programmes: current practice and future research reflections. Appl. Health Econ. Health Policy 2 (1), 55-64.

Ryan, M., Krucien, N., Hermens, F., 2018. The eyes have it: Using eye tracking to inform information processing strategies in multi-attributes choices. Health Econ. 27 (4), 709-721.

Sarrias, M., et al., 2016. Discrete choice models with random parameters in R: The rchoice package. J. Stat. Softw. 74 (10), 1-31.

Schold, J.D., Meier-Kriesche, H.-U., 2006. Which renal transplant candidates should accept marginal kidneys in exchange for a shorter waiting time on dialysis? Clin. J. Am. Soc. Nephrol. 1 (3), 532-538.

Sirchia, G., Poli, F., Cardillo, M., Scalamogna, M., Rebulla, P., Taioli, E., Remuzzi, G., Nocera, A., 1998. Cadaver kidney allocation in the north Italy transplant program on the eve of the new millennium. Clin. Transpl., 133-145.

Sonnier, G., Ainslie, A., Otter, T., 2007. Heterogeneity distributions of willingness-to-pay in choice models. Quant. Market. Econ. 5 (3), 313-331.

Sunjae Bae, A., Massie, A., Thomas, e.a., 2019. Who can tolerate a marginal kidney? Predicting survival after deceased donor kidney transplant by donor-recipient combination. Am. J. Transpl. 19 (2), 425-433.

Train, K., Weeks, M., 2005. Discrete choice models in preference space and willingness-to-pay space. In: Applications of Simulation Methods in Environmental and Resource Economics., pp. 1-16.

Venettoni, S., Grigioni, W., Grossi, P., Gianelli, C.A., Nanni, C.A., 2006. Criteria and terms for certified suitability of organ donors: assumptions and operational strategies in Italy. Annali dell'Istituto superiore di sanità 43 (3), 279-286. 